\title{
ON THE LOCAL EXISTENCE OF ANALYTIC SOLUTIONS TO THE PRANDTL BOUNDARY LAYER EQUATIONS*
}

\author{
IGOR KUKAVICA ${ }^{\dagger}$ AND VLAD VICOL ${ }^{\ddagger}$
}

\begin{abstract}
We address the local well-posedness of the Prandtl boundary layer equations. Using a new change of variables we allow for more general data than previously considered, that is, we require the matching at the top of the boundary layer to be at a polynomial rather than exponential rate. The proof is direct, via analytic energy estimates in the tangential variables.
\end{abstract}

Key words. Boundary layer, Prandtl equation, well-posedness, real-analyticity, matched asymptotics, inviscid limit.

AMS subject classifications. 35Q35, 76N10, 76N20.

\section{Introduction}

The inviscid limit of the Navier-Stokes equations on a domain $\Omega \in \mathbb{R}^{d}$ with a boundary is one of the most challenging problems in mathematical fluid mechanics (cf. $[5,6,17,18,24,25,26,34,35]$ and references therein). Near the boundary of the domain, the main difficulty is due to the incompatibility in boundary conditions between the Navier-Stokes equations $\left(u^{N S}=0\right.$ on $\left.\partial \Omega\right)$ and the Euler equations $\left(u^{E}\right.$. $n=0$ on $\partial \Omega$, where $n$ is the outward unit normal), giving rise to the boundary layer separation. The foundations for the boundary layer theory were laid by Prandtl, who in [30] made the ansatz $u^{N S}(x, \tilde{y}, t)=(u(x, \tilde{y} / \sqrt{\nu}, t), \sqrt{\nu} w(x, \tilde{y} / \sqrt{\nu}, t))$. Inserting this velocity field in the Navier-Stokes equations and sending the kinematic viscosity $\nu$ to zero, one formally obtains the Prandtl boundary layer equations for the unknown velocity field $(u, \sqrt{\nu} w)$,

$$
\begin{aligned}
& \partial_{t} u-\partial_{Y Y} u+u \partial_{x} u+w \partial_{Y} u+\partial_{x} P=0, \\
& \partial_{x} u+\partial_{Y} w=0, \\
& \partial_{Y} P=0,
\end{aligned}
$$

in $\mathbb{H}=\left\{(x, Y) \in \mathbb{R}^{2}: Y>0\right\}$, where $Y=\tilde{y} / \sqrt{\nu}$ is the normal variable in the boundary layer. For details on the formal derivation of the Prandtl boundary layer equations we refer the reader to $[4,12,9,29,30]$ and references therein. For simplicity of the presentation, in this paper we consider the two-dimensional setting, but all the methods and results presented here extend to the three-dimensional case as well (cf. Remark 3.3 below). The system (1.1)-(1.3) is supplemented with the no-slip and the no-influx boundary conditions

$$
\begin{aligned}
& \left.u(x, Y, t)\right|_{Y=0}=0, \\
& \left.w(x, Y, t)\right|_{Y=0}=0,
\end{aligned}
$$

*Received: November 30, 2011; accepted (in revised form): May 8, 2012. Communicated by Lenya Ryzhik.

${ }^{\dagger}$ Department of Mathematics, University of Southern California, 3620 S. Vermont Avenue, Los Angeles, CA 90089, USA (kukavica@usc.edu).

IK was supported in part by the NSF grant DMS-1009769.

${ }^{\ddagger}$ Department of Mathematics, University of Chicago, 5734 University Avenue, Chicago, IL 60637, USA (vicol@math.uchicago.edu).

VV Was supported in part by the NSF grant DMS-1211828. 
for $t>0$, and the matching conditions with the Euler flow as $Y \rightarrow \infty$, via the Bernoulli law

$$
\begin{aligned}
& \lim _{Y \rightarrow \infty} u(x, Y, t)=U(x, t), \\
& \partial_{x} P(x, t)=-\left(\partial_{t}+U(x, t) \partial_{x}\right) U(x, t),
\end{aligned}
$$

for $x \in \mathbb{R}^{2}, t>0$, where $U(x, t)$ is the trace at $\tilde{y}=0$ of the tangential component of the Euler flow $u^{E}$. Note that (1.3) and (1.7) determine $\partial_{x} P$ on $\mathbb{H}$. The Prandtl equations (1.1)-(1.7) are supplemented with an initial condition

$$
\left.u(x, Y, t)\right|_{t=0}=u_{0}(x, Y)
$$

in $\mathbb{H}$, which satisfies the compatibility condition with the Euler flow and the compatibility arising from the boundary condition

$$
u_{0}(x, Y=0)=0, \lim _{Y \rightarrow \infty} u_{0}(x, Y)=\left.U(x, t)\right|_{t=0} .
$$

From the mathematical point of view, the two basic problems that have to be addressed for (1.1)-(1.8) are the well/ill-posedness of the equations (cf. [8, 10, 11, 12, $16,23,28,31,37]$ ), and the rigorous justification of the singular asymptotics through which the equations were derived in the inviscid limit (cf. [14, 15, 31, 32]). For a complete survey of the mathematical and numerical results on the Prandtl boundary layer equations, we refer the reader to $[4,9,11,29]$.

In this paper we address the issue of local well-posedness of the Prandtl boundary layer equations. In establishing such results the main difficulty is to control the loss of one $x$-derivative in the convection terms of (1.1). Whether such a control is possible depends on the functional space in which we work in. In the general Sobolevspace setting, the example of [10] exhibits a solution which develops a singularity in finite time. Additionally, in $[12,15]$ it is proven that if one linearizes the system (1.1)-(1.7) about certain unstable flows, the resulting linear equations are ill-posed in Sobolev spaces. These strong instability results were used in [13] to prove that the full, nonlinear equations cannot give rise to a Lipschitz continuous semigroup in Sobolev spaces, suggesting that in order to obtain a well-posed problem one needs to work either in function spaces that impose either more structure on the evolution, or in spaces which are smoother than the Sobolev spaces.

The available well-posedness results for the Prandtl boundary layer equations fall in one of the two categories: monotonicity in the $Y$ variable (cf. $[28,29,37]$ ), or analyticity in the $x$ variable, Sobolev regularity in the $y$ variable, and exponential decay of $u(x, Y, t)-U(x, t)$ as $Y \rightarrow \infty$ (cf. [23,31,32]). Besides the local well-posedness of (1.1)-(1.7) in the analytic setting, Sammartino and Caflisch [31, 32] also establish (by using the abstract Cauchy-Kowalewski Theorem [1]) the convergence of the NavierStokes solution to the Euler solution plus the Prandtl solution in the inviscid limit, locally in time, thereby fully justifying the Prandtl equations in this setting.

The main result of this paper, Theorem 3.1 below, is to give a new local wellposedness theorem in spaces of real-analytic functions for the Prandtl boundary layer equations. The main improvement is that our proof does not require that $u(x, Y, t)-U(x, t)$ decays exponentially as $Y \rightarrow \infty$, which is not physically justified. Instead, we only require that the matching between the Prandtl solution and the Euler solution, at the top of the boundary layer, is given by a power law $1 / Y^{1+\epsilon}$ for some $\epsilon>0$. This is achieved by a suitable dynamic change of variables in the normal 
coordinate (cf. (2.4)-(2.5) below). At the cost of studying an elliptic problem (in $Y$ ) with variable coefficients (in $x$ ), in the reformulated Prandtl equations (2.6)-(2.10) the linear $Y$ growth due to the boundary condition at the top of the boundary layer is moved from the linear term onto the nonlinearity, which has faster decay in $Y$ since it is quadratic. Our proof is elementary, via higher order energy estimates, and does not rely on the abstract Cauchy-Kowalewski Theorem. The analytic norm which we use in this paper has a direct analogue for the Euler and Navier-Stokes equations (cf. $[21,22]$ ), making this proof amenable for studying the inviscid limit for solutions which are analytic in $x$ and decay algebraically in $Y$. We emphasize that analyticity is a suitable tool for studying the physics of the inviscid limit via asymptotic expansions, as was shown in the classical works of Sammartino and Caflisch [31, 32].

From the physical point of view a classical quantity in boundary layer theory is the displacement thickness, which quantifies the effect of the boundary layer on the Euler flow $u^{E}$, and is defined as (cf. $\left.[3,7,33]\right)$

$$
\delta_{1}(x)=\int_{0}^{\infty}\left(1-\frac{u(x, Y)}{U(x)}\right) d Y .
$$

More precisely, $\delta_{1}$ is the "distance through which streamlines just outside the boundary layer are displaced laterally by the retardation of fluid in the boundary layer" (cf. Batchelor [3, p. 311]). Therefore, the polynomial matching of order $1 / Y^{1+\epsilon}$ (for any $\epsilon>0$ ) considered in this paper is sharp, as any slower power-law decay is inconsistent with the definition of the displacement thickness (1.10). Polynomial matching at the top of the boundary layer, but for the stationary problem and for Oleinik-type data, was also considered in $[27,36]$.

The paper is organized as follows. In Section 2 we derive an equivalent formulation of the Prandtl equations (more details are given in Appendix A). In Section 3 we introduce the functional framework of the paper, state the main result, Theorem 3.1, and give the a priori estimates needed to prove it assuming some a priori bounds on the nonlinear, linear, and force terms appearing in (2.6) below. Sections 4, 5, and 6 are devoted to the proof of lemmas $3.1,3.2$, and 3.3 respectively.

\section{Reformulation of the Prandtl boundary layer equations}

In this section we introduce a change of variables $Y \mapsto y$ and $u \mapsto v$ which homogenizes the boundary condition at $Y=\infty$ and removes the resulting high order terms. Denote by $A(x, t)$ the unique real-analytic solution of the initial value problem

$$
\begin{aligned}
& \partial_{t} A(x, t)+U(x, t) \partial_{x} A(x, t)=A(x, t) \partial_{x} U(x, t), \\
& \left.A(x, t)\right|_{t=0}=1
\end{aligned}
$$

on $\mathbb{R} \times[0, T]$, for some $T>0$. The existence and uniqueness (in the class of real analytic functions) of $A(x, t)$ on $[0, T]$ follows from the classical Cauchy-Kowalewski Theorem since the functions $U(x, t)$ and $\partial_{x} U(x, t)$ are assumed to be uniformly real-analytic on $[0, T]$, with radius of analyticity bounded from below by some $\tau_{E}>0$. By possibly reducing $T$ we may assume that $1 / 2 \leq A(x, t) \leq 2$ on $\mathbb{R} \times[0, T]$ (this is possible since $U$ and $\partial_{x} U$ belong to $\left.L^{\infty}(\mathbb{R} \times[0, T])\right)$. Let the uniform radius of real-analyticity of the function $A(x, t)$ be bounded from below by some $\tau_{*}>0$, which depends on $\tau_{E}$ and the analytic norm on $U$ and $\partial_{x} U$ on $[0, T]$. It is convenient to introduce the real-analytic function

$$
a(x, t)=\log A(x, t),
$$


so that we have $\partial_{x} A / A=\partial_{x} a$. Define the functions

$$
\phi(y)=\langle y\rangle^{-\theta}
$$

and

$$
\Phi(y)=\int_{0}^{y} \phi(\zeta) d \zeta
$$

where

$$
\langle y\rangle=\sqrt{1+y^{2}},
$$

and $\theta>0$ is a parameter to be determined. We make the change of variables

$$
\begin{aligned}
y & =Y A(x, t), \\
v(x, y, t) & =u(x, Y, t)-(1-\phi(y)) U(x, t) .
\end{aligned}
$$

Under this change of variables, the Prandtl system (1.1)-(1.8) reads

$$
\partial_{t} v-A^{2} \partial_{y y} v+N(v)+L(v)=F,
$$

where we denoted

$$
\begin{aligned}
& \quad \begin{array}{l}
N(v)=v \partial_{x} v-\partial_{x} W(v) \partial_{y} v+\partial_{x} a W(v) \partial_{y} v \\
W(v)(x, y)=\int_{0}^{y} v(x, \zeta) d \zeta
\end{array} \\
& \begin{array}{r}
L(v)=\partial_{x} W(v) \partial_{y} \phi U \\
\quad+\partial_{x} v(1-\phi) U+\partial_{y} v\left(\Phi \partial_{x} U-\partial_{x} a \Phi U\right)-W(v) \partial_{x} a \partial_{y} \phi U+v(1-\phi) \partial_{x} U
\end{array} \\
& F=\left(\phi(1-\phi)+\Phi \partial_{y} \phi\right) U \partial_{x} U-\partial_{x} a \partial_{y} \phi \Phi U^{2}-A^{2} \partial_{y y} \phi U-\phi \partial_{x} P
\end{aligned}
$$

(cf. Appendix A for details). The system (2.6)-(2.10) is supplemented with the boundary conditions

$$
\begin{aligned}
& \left.v(x, y, t)\right|_{y=0}=\left.u(x, Y, t)\right|_{Y=0}-(1-\phi(0)) U(x, t)=0, \\
& \lim _{y \rightarrow \infty} v(x, y, t)=\lim _{Y \rightarrow \infty} u(x, Y, t)-U(x, t)=0,
\end{aligned}
$$

for all $(x, t) \in \mathbb{R} \times[0, \infty)$, and the initial condition

$$
\left.v(x, y, t)\right|_{t=0}=v_{0}(x, y)=u_{0}(x, Y)-(1-\phi(y)) U_{0}(x) .
$$

The initial datum is assumed to satisfy the compatibility conditions arising from (2.11)-(2.12), and hence

$$
\begin{aligned}
& \left.v_{0}(x, y)\right|_{y=0}=0, \\
& \lim _{y \rightarrow \infty} v_{0}(x, y)=0 .
\end{aligned}
$$

Moreover, we assume that $v_{0}$ is analytic in the $x$ variable, with uniform radius of analyticity at least $\tau_{0}>0$. Analyticity in $x$ is also assumed for the trace of the tangential component of the Euler flow $U(x, t)$, and the pressure of the Euler flow $P(x, t)$. In the two-dimensional case, if the initial data for the Euler flow is real-analytic, it remains real-analytic for all time (cf. $[2,20,21,22]$ ), and its radius of analyticity at time $t$ is bounded from below by $C \exp (-\exp (C t))$, for some positive constant $C$ depending on the initial data. In the three-dimensional case the persistence of real-analyticity holds (cf. $[2,21,22]$ ), i.e., a solution remains analytic as long as it is regular. 


\section{A priori estimates}

We shall consider the $x$-analytic norm with $y$-weight given by

$$
\rho(y)=\langle y\rangle^{\alpha}
$$

for some $\alpha>0$ to be fixed later. Namely, for a function $V(x, y)$ and a number $\tau_{0}>0$ we denote

$$
\|V\|_{X_{\tau_{0}}}^{2}=\sum_{m \geq 0}\left\|\rho(y) \partial_{x}^{m} V(x, y)\right\|_{L^{2}(\mathbb{H})}^{2} \tau_{0}^{2 m} M_{m}^{2}
$$

where the analytic weights $M_{m}$ are defined as

$$
M_{m}=\frac{(m+1)^{r}}{m !}
$$

for some $r>0$ to be determined. If $v(x, y, t)$ and $\tau(t)$ have $t$-dependence, we similarly denote

$$
\|v(t)\|_{X_{\tau(t)}}^{2}=\sum_{m \geq 0}\left\|\rho(y) \partial_{x}^{m} v(x, y, t)\right\|_{L^{2}(\mathbb{H})}^{2} \tau(t)^{2 m} M_{m}^{2} .
$$

If the $t$ dependence is clear from the context we will omit it. Since the weight $\rho(y)$ does not depend on $x$, the analytic norm may also be written as

$$
\|v\|_{X_{\tau}}^{2}=\sum_{m \geq 0}\|\rho v\|_{\dot{H}_{x}^{m}}^{2} \tau^{2 m} M_{m}^{2}
$$

For a positive number $\tau>0$ we write $v \in X_{\tau}$ if $\|v\|_{X_{\tau}}<\infty$. The main result of this paper is the following theorem.

TheOREm 3.1. Fix real numbers $\alpha>1 / 2, \theta>\alpha+1 / 2$, and $r>1$. Assume that the initial datum for the underlying Euler flow is uniformly real analytic, with radius of analyticity at least $\tau_{E}>0$ and analytic norm bounded by $G_{E}>0$. There exists $\tau_{0}=$ $\tau_{0}\left(r, \tau_{E}, G_{E}\right)>0$ such that for all $v_{0} \in X_{\tau_{0}}$ there is $T_{*}=T_{*}\left(r, \alpha, \theta, \tau_{E}, G_{E}, \tau_{0},\left\|v_{0}\right\|_{X_{\tau_{0}}}\right)>$ 0 such that the initial value problem (2.6)-(2.13) has a unique real-analytic solution on $\left[0, T_{*}\right]$.

REMARK 3.2. The above theorem shows that solutions may be constructed even if the initial datum $v_{0}$ decays only as $\langle y\rangle^{-\alpha-1 / 2-\epsilon}$ for arbitrary $\alpha>1 / 2$ and $\epsilon>0$, so that $v_{0}\langle y\rangle^{\alpha} \in L_{y}^{2}([0, \infty))$. This improves on the previous works [12, 23, 31, 32] which require the initial data for the Prandtl system to match the underlying Euler flow exponentially at $Y=\infty$.

REMARK 3.3. Although we have stated the main theorem in two dimensions, i.e. $x \in \mathbb{R}$, the same theorem holds in the three-dimensional case $x \in \mathbb{R}^{2}$. The relevant modifications needed are to redefine $\|v\|_{X_{\tau}}^{2}$ as

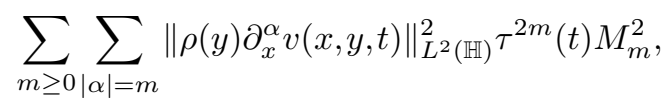

and to let the function $A(x, t)$ from the change of variables (2.4) solve the PDE

$$
\partial_{t} A(x, t)+\left(U(x, t) \cdot \nabla_{x}\right) A(x, t)=\left(A(x, t) \cdot \nabla_{x}\right) U(x, t) .
$$


Other modifications, such as the different exponents in Agmon's inequality, are straightforward.

REMARK 3.4. The proof consists of a priori estimates which can be made formal via the standard Picard iteration procedure. This is possible since we are working in spaces of real-analytic functions.

We now turn to the a priori estimates needed to prove Theorem 3.1. From the Definition (3.2), we have formally

$$
\frac{1}{2} \frac{d}{d t}\|v\|_{X_{\tau}}^{2}+(-\dot{\tau})\|v\|_{Y_{\tau}}^{2}=\sum_{m \geq 0}\left(\frac{1}{2} \frac{d}{d t}\|\rho v\|_{\dot{H}_{x}^{m}}^{2}\right) \tau^{2 m} M_{m}^{2},
$$

where we omitted the time time dependence of $v$ and $\tau$, and have denoted

$$
\|v\|_{Y_{\tau}}^{2}=\sum_{m \geq 1}\|\rho v\|_{\dot{H}_{x}^{m}}^{2} \tau^{2 m-1} m M_{m}^{2}
$$

The heart of the matter consists of estimating the term on the right side of (3.3) via Sobolev energy estimates. We fix $m \geq 0$, apply $\partial_{x}^{m}$ to (2.6), multiply the resulting equality by $\rho^{2} \partial_{x}^{m} v$, and integrate it over the half plane to obtain

$$
\frac{1}{2} \frac{d}{d t}\left\|\rho \partial_{x}^{m} v\right\|_{L^{2}}^{2}-\left\langle\partial_{x}^{m}\left(A^{2} \partial_{y y} v\right), \rho^{2} \partial_{x}^{m} v\right\rangle=\left\langle\rho \partial_{x}^{m}(F-N(v)-L(v)), \rho \partial_{x}^{m} v\right\rangle .
$$

In the dissipative term we use the Leibniz rule to distribute the derivative in $x$, and then integrate by parts in $y$ to obtain

$$
\begin{aligned}
& \frac{1}{2} \frac{d}{d t}\left\|\rho \partial_{x}^{m} v\right\|_{L^{2}}^{2}+\left\|A \rho \partial_{y} \partial_{x}^{m} v\right\|_{L^{2}}^{2} \\
= & -2\left\langle A \partial_{y} \rho \partial_{x}^{m} v, A \rho \partial_{y} \partial_{x}^{m} v\right\rangle-\sum_{j=1}^{m}\left(\begin{array}{c}
m \\
j
\end{array}\right)\left\langle A^{-2} \partial_{x}^{j}\left(A^{2}\right) A \rho \partial_{y} \partial_{x}^{m-j} v, A \rho \partial_{y} \partial_{x}^{m} v\right\rangle \\
& \quad-2 \sum_{j=1}^{m}\left(\begin{array}{c}
m \\
j
\end{array}\right)\left\langle A^{-1} \partial_{x}^{j}\left(A^{2}\right) A \partial_{y} \rho \partial_{y} \partial_{x}^{m-j} v, \rho \partial_{x}^{m} v\right\rangle+\left\langle\rho \partial_{x}^{m}(F-N(v)-L(v)), \rho \partial_{x}^{m} v\right\rangle .
\end{aligned}
$$

The integration by parts in $y$ is formally justified by introducing a smooth cut-off function $\eta(y)$ such that $\eta \equiv 1$ on $0 \leq y \leq 1$ and $\eta \equiv 0$ on $y \geq 2$. For any $R>0$ we have (ignoring $A(x, t)$ since it doesn't depend on $y$ )

$$
\begin{aligned}
\int u \partial_{y y} u \rho(y) \eta(y / R) d y=\int & \partial_{y} u \partial_{y} u \rho(y) \eta(y / R) d y-\frac{1}{2} \int u^{2} \partial_{y y} \rho \eta(y / R) d y \\
& -\frac{1}{R} \int u^{2} \partial_{y} \rho(y) \partial_{y} \eta(y / R) d y-\frac{1}{2 R^{2}} \int u^{2} \rho(y) \partial_{y y} \eta(y / R) d y .
\end{aligned}
$$

Due to our choice of $\rho$ one may pass $R \rightarrow \infty$ in the above equation, so that the last two terms on the right side of the above identity vanish, justifying the integration by parts in (3.6). We recall that $\rho(y)=\langle y\rangle^{\alpha}$, and hence

$$
\partial_{y} \rho(y) \leq \alpha\langle y\rangle^{\alpha-1} \leq \frac{\alpha}{\langle y\rangle} \rho(y) \leq \alpha \rho(y)
$$


for all $y \geq 0$. From (3.6) and (3.7), using $\|A\|_{L^{\infty}} \leq 2$ and $\left\|A^{-1}\right\|_{L^{\infty}} \leq 2$ on $[0, T]$, we obtain

$$
\begin{aligned}
& \frac{1}{2} \frac{d}{d t}\left\|\rho \partial_{x}^{m} v\right\|_{L^{2}}^{2}+\left\|A \rho \partial_{y} \partial_{x}^{m} v\right\|_{L^{2}}^{2} \\
& \leq\left|\left\langle\rho \partial_{x}^{m}(F-N(v)-L(v)), \rho \partial_{x}^{m} v\right\rangle\right|+4 \alpha\left\|\rho \partial_{x}^{m} v\right\|_{L^{2}}\left\|A \rho \partial_{y} \partial_{x}^{m} v\right\|_{L^{2}} \\
& \quad+4 \sum_{j=1}^{m}\left(\begin{array}{c}
m \\
j
\end{array}\right)\left\|\partial_{x}^{j}\left(A^{2}\right)\right\|_{L^{\infty}}\left\|A \rho \partial_{y} \partial_{x}^{m-j} v\right\|_{L^{2}}\left\|A \rho \partial_{y} \partial_{x}^{m} v\right\|_{L^{2}} \\
& \quad+4 \alpha \sum_{j=1}^{m}\left(\begin{array}{c}
m \\
j
\end{array}\right)\left\|\partial_{x}^{j}\left(A^{2}\right)\right\|_{L^{\infty}}\left\|A \rho \partial_{y} \partial_{x}^{m-j} v\right\|_{L^{2}}\left\|\rho \partial_{x}^{m} v\right\|_{L^{2}} .
\end{aligned}
$$

Before estimating the force, linear, and nonlinear terms on the right side of (3.8), we first check that the last three terms on the right side of (3.8) can be suitably treated. Inserting the estimate (3.8) into (3.3), we obtain

$$
\begin{aligned}
& \quad \frac{1}{2} \frac{d}{d t}\|v\|_{X_{\tau}}^{2}+(-\dot{\tau})\|v\|_{Y_{\tau}}^{2}+\|v\|_{Z_{\tau}}^{2} \\
& \leq C \sum_{m \geq 0} X_{m} Z_{m}+\sum_{m \geq 0}\left|\left\langle\rho \partial_{x}^{m}(F-N(v)-L(v)), \rho \partial_{x}^{m} v\right\rangle\right| \tau^{2 m} M_{m}^{2} \\
& \quad+C \sum_{m \geq 1} \sum_{j=1}^{m}\left(\begin{array}{c}
m \\
j
\end{array}\right)\left\|\partial_{x}^{j}\left(A^{2}\right)\right\|_{L^{\infty}} Z_{m-j}\left(Z_{m}+X_{m}\right) \tau^{j} M_{m} M_{m-j}^{-1}
\end{aligned}
$$

for some dimensional constant $C>0$, where we have introduced the norm

$$
\|v\|_{Z_{\tau}}^{2}=\sum_{m \geq 0}\left\|A \rho \partial_{y} \partial_{x}^{m} v\right\|_{L^{2}}^{2} \tau^{2 m} M_{m}^{2}
$$

and

$$
\begin{aligned}
X_{m} & =\left\|\rho \partial_{x}^{m} v\right\|_{L^{2}} \tau^{m} M_{m} \\
Y_{m} & =\left\|\rho \partial_{x}^{m} v\right\|_{L^{2}} \tau^{m-1 / 2} m^{1 / 2} M_{m} \\
Z_{m} & =\left\|A \rho \partial_{y} \partial_{x}^{m} v\right\|_{L^{2}} \tau^{m} M_{m}
\end{aligned}
$$

so that we have, conveniently,

$$
\begin{gathered}
\|v\|_{X_{\tau}}^{2}=\sum_{m \geq 0} X_{m}^{2}, \\
\|v\|_{Y_{\tau}}^{2}=\sum_{m \geq 1} Y_{m}^{2}, \\
\|v\|_{Z_{\tau}}^{2}=\sum_{m \geq 0} Z_{m}^{2} .
\end{gathered}
$$

In order to estimate $\partial_{x}^{j}\left(A^{2}\right)$ we assume that the Euler flow $U$ does not blow up on $[0, T]$, and that $U(x, 0)$ is a real-analytic function of $x$. As a consequence of these two assumptions we have that for all $t \in[0, T]$ the analyticity radius of the functions $U(\cdot, t)$ and $\partial_{x} U(\cdot, t)$ are bounded from below by some strictly positive constant $\tau_{E}$ (cf. $[2,21,22]$ ). More precisely, there exists a constant $G_{E} \geq 1$ such that

$$
\left\|\partial_{x}^{j} U\right\|_{L^{\infty}\left([0, T] ; L^{\infty}\right)}+\left\|\partial_{x}^{j}\left(\partial_{x} U\right)\right\|_{L^{\infty}\left([0, T] ; L^{\infty}\right)} \leq G_{E} \frac{j !}{\tau_{E}^{j}}
$$


for all $j \geq 0$. From the Cauchy-Kowalewski Theorem we have that there exists $0<$ $\tau_{*}=\tau_{*}\left(\tau_{E}, M, r\right) \leq \tau_{E} / 2$ and $G \geq 2 G_{E}$ such that

$$
\left\|\partial_{x}^{j}\left(A^{2}\right)\right\|_{L^{\infty}\left([0, T] ; L^{\infty}\right)} \leq G \frac{1}{\tau_{*}^{j} M_{j}}
$$

for all $j \geq 0$, where $M_{j}$ is as defined in (3.1). In fact, by possibly decreasing $\tau_{*}$ and increasing $G$ we may also assume that

$$
\left\|\partial_{x}^{j} a\right\|_{L^{\infty}\left([0, T] ; L^{\infty}\right)} \leq G \frac{1}{\tau_{*}^{j} M_{j}}
$$

for all $j \geq 0$. Therefore, inserting (3.17) into (3.9) we obtain

$$
\begin{gathered}
\quad \frac{1}{2} \frac{d}{d t}\|v\|_{X_{\tau}}^{2}+(-\dot{\tau})\|v\|_{Y_{\tau}}^{2}+\|v\|_{Z_{\tau}}^{2} \\
\leq C \sum_{m \geq 0} X_{m} Z_{m}+\sum_{m \geq 0}\left|\left\langle\rho \partial_{x}^{m}(F-N(v)-L(v)), \rho \partial_{x}^{m} v\right\rangle\right| \tau^{2 m} M_{m}^{2} \\
\quad+C G \sum_{m \geq 1} \sum_{j=1}^{m}\left(\begin{array}{c}
m \\
j
\end{array}\right) \frac{1}{\tau_{*}^{j} M_{j}} Z_{m-j}\left(Z_{m}+X_{m}\right) \tau^{j} M_{m} M_{m-j}^{-1} \\
\leq C\|v\|_{X_{\tau}}\|v\|_{Z_{\tau}}+\sum_{m \geq 0}\left|\left\langle\rho \partial_{x}^{m}(F-N(v)-L(v)), \rho \partial_{x}^{m} v\right\rangle\right| \tau^{2 m} M_{m}^{2} \\
\quad+C G \sum_{m \geq 1} \sum_{j=1}^{m}\left(\frac{\tau}{\tau_{*}}\right)^{j} Z_{m-j}\left(Z_{m}+X_{m}\right) \frac{m ! M_{m}}{j ! M_{j}(m-j) ! M_{m-j}} .
\end{gathered}
$$

Note that by (3.1) we have

$$
\frac{m ! M_{m}}{j ! M_{j}(m-j) ! M_{m-j}}=\frac{(m+1)^{r}}{(j+1)^{r}(m-j+1)^{r}} \leq C
$$

for all $0 \leq j \leq m$, for some sufficiently large constant $C=C(r)>0$. Therefore, using the discrete Young inequality

$$
\|f \cdot(g * h)\|_{\ell^{1}} \leq\|f\|_{\ell^{2}}\|g\|_{\ell^{1}}\|h\|_{\ell^{2}},
$$

and the assumption that $\tau(t)$ is decreasing, with $\tau(0)=\tau_{0} \leq \tau_{*} / 2$, we obtain

$$
\begin{gathered}
\frac{1}{2} \frac{d}{d t}\|v\|_{X_{\tau}}^{2}+\|v\|_{Z_{\tau}}^{2} \leq C \\
\|v\|_{X_{\tau}}\|v\|_{Z_{\tau}}+\sum_{m \geq 0}\left|\left\langle\rho \partial_{x}^{m}(F-N(v)-L(v)), \rho \partial_{x}^{m} v\right\rangle\right| \tau^{2 m} M_{m}^{2} \\
+C G\|v\|_{Z_{\tau}}\left(\|v\|_{Z_{\tau}}+\|v\|_{X_{\tau}}\right) \sum_{j \geq 1}\left(\frac{\tau_{0}}{\tau_{*}}\right)^{j} \\
\leq C\|v\|_{X_{\tau}}\|v\|_{Z_{\tau}}+\sum_{m \geq 0}\left|\left\langle\rho \partial_{x}^{m}(F-N(v)-L(v)), \rho \partial_{x}^{m} v\right\rangle\right| \tau^{2 m} M_{m}^{2} \\
+C_{0} G \frac{\tau_{0}}{\tau_{*}-\tau_{0}}\|v\|_{Z_{\tau}}\left(\|v\|_{Z_{\tau}}+\|v\|_{X_{\tau}}\right)
\end{gathered}
$$

for some fixed sufficiently large positive constant $C_{0}=C_{0}(r)$. Therefore, in order to absorb the $\|v\|_{Z_{\tau}}^{2}$ term on the right side of the equation, we choose $\tau_{0}=\tau_{0}\left(r, \tau_{*}, G, C_{0}\right)=$ $\tau_{0}\left(r, \tau_{E}, G_{E}, C_{0}\right)>0$ such that

$$
C_{0} G \frac{\tau_{0}}{\tau_{*}-\tau_{0}} \leq \frac{1}{4}
$$


that is $\tau_{0} \leq \tau_{*} /\left(1+4 C_{0} G\right)$, and we immediately obtain from (3.20) and the CauchySchwarz inequality that

$$
\begin{aligned}
& \frac{1}{2} \frac{d}{d t}\|v\|_{X_{\tau}}^{2}+(-\dot{\tau})\|v\|_{Y_{\tau}}^{2}+\frac{1}{2}\|v\|_{Z_{\tau}}^{2} \\
\leq & C_{1}\|v\|_{X_{\tau}}^{2}+\sum_{m \geq 0}\left|\left\langle\rho \partial_{x}^{m}(F-N(v)-L(v)), \rho \partial_{x}^{m} v\right\rangle\right| \tau^{2 m} M_{m}^{2},
\end{aligned}
$$

for some positive constant $C_{1}=C_{1}(r)$. It is left to find suitable bounds for the right side of (3.22). To this end we have the following lemmas.

Lemma 3.1. Let $\alpha>1 / 2$. We have the estimate

$$
\mathcal{N}=\sum_{m \geq 0}\left|\left\langle\rho \partial_{x}^{m}(N(v)), \rho \partial_{x}^{m} v\right\rangle\right| \tau^{2 m} M_{m}^{2} \leq C \tau^{-1}\|v\|_{Z_{\tau}}\left(\|v\|_{X_{\tau}}^{2}+\|v\|_{Y_{\tau}}^{2}\right)
$$

for some positive constant $C$ depending on $r>1$, the underlying Euler flow, and $\tau_{0}$.

Lemma 3.2. Let $\alpha>1 / 2$ and $\theta>1$ be such that $\theta>\alpha-1 / 2$. We have the bound

$$
\mathcal{L}=\sum_{m \geq 0}\left|\left\langle\rho \partial_{x}^{m}(L(v)), \rho \partial_{x}^{m} v\right\rangle\right| \tau^{2 m} M_{m}^{2} \leq C \tau^{-1}\|v\|_{X_{\tau}}\left(\|v\|_{X_{\tau}}+\|v\|_{Z_{\tau}}\right)+C\|v\|_{Y_{\tau}}^{2}
$$

for some sufficiently large positive constant $C$ depending on $r$, the analyticity radius, and the analytic norm of the underlying Euler flow.

Lemma 3.3. Let $\alpha>1 / 2$ and $\theta>1$ be such that $\theta>\alpha+1 / 2$. We have the bound

$$
\mathcal{F}=\sum_{m \geq 0}\left|\left\langle\rho \partial_{x}^{m} F, \rho \partial_{x}^{m} v\right\rangle\right| \tau^{2 m} M_{m}^{2} \leq C\|v\|_{X_{\tau}}
$$

for a suitable constant $C>0$ depending on the underlying Euler flow, $\alpha$, and $\theta$.

The proofs of lemmas 3.1, 3.2, and 3.3 are given in sections 4, 5, and 6 below. Assuming that the above three lemmas hold, we may conclude the a priori estimates needed to prove the local existence of solutions to (2.6)-(2.10). From (3.22)-(3.25) and the Cauchy-Schwarz inequality we obtain

$$
\begin{aligned}
& \frac{1}{2} \frac{d}{d t}\|v\|_{X_{\tau}}^{2}+(-\dot{\tau})\|v\|_{Y_{\tau}}^{2}+\frac{1}{2}\|v\|_{Z_{\tau}}^{2} \\
\leq & C_{*}\left(1+\tau^{-2}\right)\|v\|_{X_{\tau}}^{2}+C_{*} \tau^{-1}\|v\|_{Z_{\tau}}\|v\|_{X_{\tau}}^{2}+C_{*}\|v\|_{X_{\tau}}+C_{*}\|v\|_{Y_{\tau}}^{2}+C_{*} \tau^{-1}\|v\|_{Z_{\tau}}\|v\|_{Y_{\tau}}^{2}
\end{aligned}
$$

for some positive constant $C_{*}$ depending on the underlying Euler flow, $\alpha$, and $\theta$. Let the analyticity radius $\tau(t)$ satisfy the differential inequality

$$
\dot{\tau}+2 C_{*}+2 C_{*} \tau^{-1}\|v\|_{Z_{\tau}} \leq 0,
$$

with initial data $\tau(0)=\tau_{0}$. In particular it suffices to let

$$
\frac{d}{d t}\left(\tau^{2}\right)+4 C_{*} \tau_{0}+4 C_{*}\|v\|_{Z_{\tau}}=0
$$

which gives 


$$
\begin{aligned}
\tau(t)^{2} & =\tau_{0}^{2}-4 C_{*} \tau_{0} t-4 C_{*} \int_{0}^{t}\|v(s)\|_{Z_{\tau(s)}} d s \\
& \geq \tau_{0}^{2}-4 C_{*} \tau_{0} t-4 C_{*} t^{1 / 2}\left(\int_{0}^{t}\|v(s)\|_{Z_{\tau(s)}}^{2} d s\right)^{1 / 2} .
\end{aligned}
$$

The above estimate shows that at least for some short time $T_{*}=T_{*}\left(C_{*}, \tau_{0},\left\|v_{0}\right\|_{X_{\tau_{0}}}\right)$ we have $\tau(t)>\tau_{0} / 4$ on $\left[0, T_{*}\right]$, since by combining (3.26) with (3.27) we have

$$
\begin{aligned}
\frac{1}{2} \frac{d}{d t}\|v\|_{X_{\tau}}^{2}+\frac{1}{2}\|v\|_{Z_{\tau}}^{2} & \leq C_{*}\left(1+16 \tau_{0}^{-2}\right)\|v\|_{X_{\tau}}^{2}+4 C_{*} \tau_{0}^{-1}\|v\|_{Z_{\tau}}\|v\|_{X_{\tau}}^{2}+C_{*}\|v\|_{X_{\tau}} \\
& \leq C_{*}\left(1+16 \tau_{0}^{-2}\right)\|v\|_{X_{\tau}}^{2}+\frac{1}{4}\|v\|_{Z_{\tau}}^{2}+16 C_{*}^{2} \tau_{0}^{-2}\|v\|_{X_{\tau}}^{4}+C_{*}\|v\|_{X_{\tau}}
\end{aligned}
$$

on $\left[0, T_{*}\right]$, and therefore, if $T_{*}$ is chosen sufficiently small, we have

$$
\int_{0}^{t}\|v(s)\|_{Z_{\tau(s)}}^{2} d s \leq 2\left\|v_{0}\right\|_{X_{\tau_{0}}} .
$$

This concludes the proof of the a priori estimates, proving Theorem 3.1.

\section{The bound of the nonlinear term}

In this section we prove Lemma 3.1. We first write the nonlinear term as

$$
\mathcal{N}=\sum_{m \geq 0}\left|\left\langle\rho \partial_{x}^{m} N(v), \rho \partial_{x}^{m} v\right\rangle\right| \tau^{2 m} M_{m}^{2} \leq \mathcal{N}_{0}+\mathcal{N}_{1}+\mathcal{N}_{2}+\mathcal{N}_{3},
$$

where

$$
\begin{aligned}
& \mathcal{N}_{0}=|\langle\rho N(v), \rho v\rangle| \\
& \mathcal{N}_{1}=\sum_{m \geq 1}\left|\left\langle\rho \partial_{x}^{m}\left(v \partial_{x} v\right), \rho \partial_{x}^{m} v\right\rangle\right| \tau^{2 m} M_{m}^{2} \\
& \mathcal{N}_{2}=\sum_{m \geq 1}\left|\left\langle\rho \partial_{x}^{m}\left(\partial_{x} W(v) \partial_{y} v\right), \rho \partial_{x}^{m} v\right\rangle\right| \tau^{2 m} M_{m}^{2} \\
& \mathcal{N}_{3}=\sum_{m \geq 1}\left|\left\langle\rho \partial_{x}^{m}\left(\partial_{x} a W(v) \partial_{y} v\right), \rho \partial_{x}^{m} v\right\rangle\right| \tau^{2 m} M_{m}^{2} .
\end{aligned}
$$

The following lemmas shall be used throughout the rest of the paper.

Lemma 4.1. With $\rho(y)=\langle y\rangle^{\alpha}$, where $\alpha>1 / 2$, we have the bound

$$
\left\|\partial_{x}^{k} v\right\|_{L_{y}^{\infty} L_{x}^{2}} \leq C\left\|A \rho \partial_{y} \partial_{x}^{k} v\right\|_{L_{x, y}^{2}}
$$

for all integers $k \geq 0$, all $t \in[0, T]$, and some positive constant $C=C(\alpha)$.

Proof. For any $k \geq 0$, the function $\partial_{x}^{k} v$ vanishes at $y=0$, and thus

$$
\partial_{x}^{k} v(x, y)=\int_{0}^{y} \partial_{y} \partial_{x}^{k} v(x, \zeta) d \zeta=\frac{1}{A(x)} \int_{0}^{y} A(x) \rho(\zeta) \partial_{y} \partial_{x}^{k} v(x, \zeta) \rho(\zeta)^{-1} d \zeta .
$$


Taking the $L^{2}$ norm in the $x$ variable, and then the $L^{\infty}$ norm in the $y$ variable, gives

$$
\begin{aligned}
\left\|\partial_{x}^{k} v\right\|_{L_{y}^{\infty} L_{x}^{2}} & \leq\left\|A^{-1}\right\|_{L^{\infty}} \int_{0}^{\infty}\left\|A \rho(\zeta) \partial_{y} \partial_{x}^{k} v(\cdot, \zeta)\right\|_{L_{x}^{2}} \rho(\zeta)^{-1} d \zeta \\
& \leq 2\left\|A \rho \partial_{y} \partial_{x}^{k} v\right\|_{L_{x, y}^{2}}\left\|\rho^{-1}\right\|_{L_{y}^{2}},
\end{aligned}
$$

since $\left\|A^{-1}\right\|_{L^{\infty}} \leq 2$. This concludes the proof of the lemma if $\rho^{-1} \in L^{2}([0, \infty))$, which is ensured by choosing $\alpha>1 / 2$.

Using the Agmon inequality, Lemma 4.1 implies

$$
\left\|\partial_{x}^{k} v\right\|_{L_{x, y}^{\infty}} \leq C\left\|A \rho \partial_{y} \partial_{x}^{k} v\right\|_{L_{x, y}^{2}}^{1 / 2}\left\|A \rho \partial_{y} \partial_{x}^{k+1} v\right\|_{L_{x, y}^{2}}^{1 / 2}
$$

for all $k \geq 0$.

Lemma 4.2. With $\rho(y)=\langle y\rangle^{\alpha}$, where $\alpha>1 / 2$, we have the bound

$$
\left\|\partial_{x}^{k} W(v)\right\|_{L_{y}^{\infty} L_{x}^{2}} \leq C\left\|\rho \partial_{x}^{k} v\right\|_{L_{x, y}^{2}}
$$

for all integers $k \geq 0, t \in[0, T]$, where $C=C(\alpha)$ is a sufficiently large constant.

Proof. We recall from (2.8) that $W(v)(x, y)=\int_{0}^{y} v(x, \zeta) d \zeta$. As in the proof of Lemma 4.1, we estimate

$$
\begin{aligned}
\left\|\partial_{x}^{k} W(v)(\cdot, y)\right\|_{L_{x}^{2}} & \leq \int_{0}^{y}\left\|\partial_{x}^{k} v(\cdot, \zeta)\right\|_{L_{x}^{2}} d \zeta=\int_{0}^{y}\left\|\rho(\zeta) \partial_{x}^{k} v(\cdot, \zeta)\right\|_{L_{x}^{2}} \rho(\zeta)^{-1} d \zeta \\
& \leq \int_{0}^{\infty}\left\|\rho(\zeta) \partial_{x}^{k} v(\cdot, \zeta)\right\|_{L_{x}^{2}} \rho(\zeta)^{-1} d \zeta \\
& \leq\left\|\rho \partial_{x}^{k} v\right\|_{L_{x, y}^{2}}\left\|\rho^{-1}\right\|_{L_{y}^{2}}
\end{aligned}
$$

Hence, we obtain

$$
\left\|\partial_{x}^{k} W(v)\right\|_{L_{y}^{\infty} L_{x}^{2}} \leq C(\alpha)\left\|\rho \partial_{x}^{k} v\right\|_{L_{x, y}^{2}}
$$

provided $\rho^{-1} \in L^{2}([0, \infty))$. This is ensured by $\alpha>1 / 2$ and the proof of the lemma is concluded.

Using Agmon's inequality in the $x$ variable, Lemma 4.2 implies

$$
\left\|\partial_{x}^{k} W(v)\right\|_{L_{x, y}^{\infty}} \leq C\left\|\rho \partial_{x}^{k} v\right\|_{L_{x, y}^{2}}^{1 / 2}\left\|\rho \partial_{x}^{k+1} v\right\|_{L_{x, y}^{2}}^{1 / 2}
$$

for some positive constant $C$. We now proceed by estimating the four terms on the far right side of (4.1).

4.1. $\mathcal{N}_{0}$ bound. The term $\mathcal{N}_{0}$ is bounded using the Hölder inequality as

$$
\begin{gathered}
\mathcal{N}_{0} \leq\|\rho v\|_{L^{2}}\left(\left\|\rho v \partial_{x} v\right\|_{L^{2}}+\left\|\rho \partial_{x} W(v) \partial_{y} v\right\|_{L^{2}}+\left\|\rho \partial_{x} a W(v) \partial_{y} v\right\|_{L^{2}}\right) \\
\leq\|\rho v\|_{L^{2}}\left(\left\|\rho \partial_{x} v\right\|_{L^{2}}\|v\|_{L^{\infty}}+\left\|A^{-1}\right\|_{L^{\infty}}\left\|A \rho \partial_{y} v\right\|_{L^{2}}\left\|\partial_{x} W(v)\right\|_{L^{\infty}}\right. \\
\left.+\left\|A^{-1}\right\|_{L^{\infty}}\left\|\partial_{x} a\right\|_{L^{\infty}}\left\|A \rho \partial_{y} v\right\|_{L^{2}}\|W(v)\|_{L^{\infty}}\right) .
\end{gathered}
$$

We apply (4.3), (4.5), and recall the notation (3.10)-(3.12) to obtain

$$
\begin{aligned}
\mathcal{N}_{0} & \leq C \tau^{-1}\left(X_{0} Y_{1} Z_{0}^{1 / 2} Z_{1}^{1 / 2}+X_{0} Z_{0} Y_{1}^{1 / 2} Y_{2}^{1 / 2}+\tau^{3 / 4} X_{0} Z_{0} X_{0}^{1 / 2} Y_{1}^{1 / 2}\right) \\
& \leq C \tau^{-1}\left(\|v\|_{X_{\tau}}\|v\|_{Y_{\tau}}\|v\|_{Z_{\tau}}+\|v\|_{X_{\tau}}^{3 / 2}\|v\|_{Y_{\tau}}^{1 / 2}\|v\|_{Z_{\tau}}\right)
\end{aligned}
$$

for some positive constant $C=C\left(\alpha, G, \tau_{E}, \tau_{0}\right)$. 
4.2. $\mathcal{N}_{1}$ bound. In order to bound $\mathcal{N}_{1}$, we use the Leibniz rule and the Cauchy-Schwarz inequality to get

$$
\mathcal{N}_{1} \leq \sum_{m \geq 1} \sum_{j=0}^{m}\left(\begin{array}{c}
m \\
j
\end{array}\right)\left\|\rho \partial_{x}^{j} v \partial_{x}^{m-j+1} v\right\|_{L^{2}}\left\|\rho \partial_{x}^{m} v\right\|_{L^{2}} \tau^{2 m} M_{m}^{2}
$$

For $0 \leq j \leq[m / 2]$ we bound (using the Hölder inequality, (4.2), and the Agmon inequality in the $x$ variable)

$$
\begin{aligned}
\left\|\rho \partial_{x}^{j} v \partial_{x}^{m-j+1} v\right\|_{L^{2}} & \leq\left\|\partial_{x}^{j} v\right\|_{L^{\infty}}\left\|\rho \partial_{x}^{m-j+1} v\right\|_{L^{2}} \\
& \leq C\left\|A \rho \partial_{y} \partial_{x}^{j} v\right\|_{L^{2}}^{1 / 2}\left\|A \rho \partial_{y} \partial_{x}^{j+1} v\right\|_{L^{2}}^{1 / 2}\left\|\rho \partial_{x}^{m-j+1} v\right\|_{L^{2}},
\end{aligned}
$$

and similarly, for $[m / 2]+1 \leq j \leq m$, we have

$$
\begin{aligned}
\left\|\rho \partial_{x}^{j} v \partial_{x}^{m-j+1} v\right\|_{L^{2}} & \leq\left\|\rho \partial_{x}^{j} v\right\|_{L^{2}}\left\|\partial_{x}^{m-j+1} v\right\|_{L^{\infty}} \\
& \leq C\left\|\rho \partial_{x}^{j} v\right\|_{L^{2}}\left\|A \rho \partial_{y} \partial_{x}^{m-j+1} v\right\|_{L^{2}}^{1 / 2}\left\|A \rho \partial_{y} \partial_{x}^{m-j+2} v\right\|_{L^{2}}^{1 / 2},
\end{aligned}
$$

for some sufficiently large constant $C=C(\alpha, G)$. Inserting (4.8) and (4.9) into (4.7) we obtain that $\mathcal{N}_{1}$ is bounded by

$$
\begin{aligned}
C & \tau^{-1 / 2} \sum_{m \geq 1} \sum_{j=0}^{[m / 2]} Z_{j}^{1 / 2} Z_{j+1}^{1 / 2} Y_{m-j+1} Y_{m} \frac{\left(\begin{array}{c}
m \\
j
\end{array}\right) M_{m}}{M_{j}^{1 / 2} M_{j+1}^{1 / 2} M_{m-j+1}(m-j+1)^{1 / 2} m^{1 / 2}} \\
& +C \tau^{-1 / 2} \sum_{m \geq 1} \sum_{j=[m / 2]+1}^{m} Y_{j} Z_{m-j+1}^{1 / 2} Z_{m-j+2}^{1 / 2} Y_{m} \frac{\left(\begin{array}{c}
m \\
j
\end{array}\right) M_{m}}{M_{j} M_{m-j+1}^{1 / 2} M_{m-j+2}^{1 / 2}(j+1)^{1 / 2} m^{1 / 2}}
\end{aligned}
$$

Using the Definition (3.1) of the analytic weights $M_{m}$, we may bound

$$
\begin{aligned}
\left(\begin{array}{c}
m \\
j
\end{array}\right) \frac{M_{m}}{M_{j}^{1 / 2} M_{j+1}^{1 / 2} M_{m-j+1}(m-j+1)^{1 / 2} m^{1 / 2}} & \leq \frac{(m+1)^{r} j^{1 / 2}(m-j+1)}{(j+1)^{r}(m-j+1)^{r}(m-j+1)^{1 / 2} m^{1 / 2}} \\
& \leq C(j+1)^{1 / 2-r}
\end{aligned}
$$

for all $0 \leq j \leq m / 2$, and similarly

$$
\begin{aligned}
\left(\begin{array}{c}
m \\
j
\end{array}\right) \frac{M_{m}}{M_{j} M_{m-j+1}^{1 / 2} M_{m-j+2}^{1 / 2}(j+1)^{1 / 2} m^{1 / 2}} & \leq \frac{(m+1)^{r}(m-j+2)^{3 / 2}}{(j+1)^{r}(m-j+1)^{r}(j+1)^{1 / 2} m^{1 / 2}} \\
& \leq C(m-j+1)^{1 / 2-r}
\end{aligned}
$$

for all $m / 2 \leq j \leq m$, and some positive constant $C=C(r)>0$. From (4.10), (4.11), and (4.12) we obtain

$$
\begin{aligned}
& \mathcal{N}_{1} \leq C \tau^{-1 / 2} \sum_{m \geq 1} \sum_{j=0}^{[m / 2]} Z_{j}^{1 / 2} Z_{j+1}^{1 / 2} Y_{m-j+1} Y_{m}(j+1)^{1 / 2-r} \\
&+C \tau^{-1 / 2} \sum_{m \geq 1} \sum_{j=[m / 2]+1}^{m} Y_{j} Z_{m-j+1}^{1 / 2} Z_{m-j+2}^{1 / 2} Y_{m}(m-j+1)^{1 / 2-r} .
\end{aligned}
$$


Using (4.13) and the discrete Young and Hölder inequalities

$$
\|f \cdot(g * h)\|_{\ell^{1}} \leq C\|f\|_{\ell^{2}}\|g\|_{\ell^{1}}\|h\|_{\ell^{2}},
$$

with $f_{k}=Y_{k}, g_{k}=Z_{k}^{1 / 2} Z_{k+1}^{1 / 2}(k+1)^{1 / 2-r}, h_{k}=Y_{k+1}$ for the first term on the right side of (4.13) and respectively $f_{k}=Y_{k}, g_{k}=Z_{k+1}^{1 / 2} Z_{k+2}^{1 / 2}(k+1)^{1 / 2-r}, h_{k}=Y_{k}$ for the second term on the right side of (4.13), we obtain

$$
\mathcal{N}_{1} \leq C \tau^{-1 / 2}\|v\|_{Z_{\tau}}\|v\|_{Y_{\tau}}^{2},
$$

provided $r-1 / 2>1 / 2$, i.e., $r>1$, so that $\left\{k^{1 / 2-r}\right\}_{k=1}^{\infty} \in \ell^{2}$.

4.3. $\mathcal{N}_{2}$ bound. In order to bound $\mathcal{N}_{2}$, we write

$$
\begin{aligned}
\mathcal{N}_{2} \leq & \sum_{m \geq 1} \sum_{j=0}^{m}\left(\begin{array}{c}
m \\
j
\end{array}\right)\left\|\rho \partial_{x}^{j+1} W(v) \partial_{y} \partial_{x}^{m-j} v\right\|_{L^{2}}\left\|\rho \partial_{x}^{m} v\right\|_{L^{2}} \tau^{2 m} M_{m}^{2} \\
\leq & \sum_{m \geq 1} \sum_{j=0}^{[m / 2]}\left(\begin{array}{c}
m \\
j
\end{array}\right)\left\|A^{-1}\right\|_{L^{\infty}}\left\|\partial_{x}^{j+1} W(v)\right\|_{L^{\infty}}\left\|A \rho \partial_{y} \partial_{x}^{m-j} v\right\|_{L^{2}}\left\|\rho \partial_{x}^{m} v\right\|_{L^{2}} \tau^{2 m} M_{m}^{2} \\
& \quad+\sum_{m \geq 1} \sum_{j=[m / 2]+1}^{m}\left(\begin{array}{c}
m \\
j
\end{array}\right)\left\|\partial_{x}^{j+1} W(v)\right\|_{L_{y}^{\infty} L_{x}^{2}}\left\|\rho \partial_{y} \partial_{x}^{m-j} v\right\|_{L_{y}^{2} L_{x}^{\infty}}\left\|\rho \partial_{x}^{m} v\right\|_{L^{2}} \tau^{2 m} M_{m}^{2} .
\end{aligned}
$$

Using (4.5), we obtain

$$
\begin{gathered}
\mathcal{N}_{2} \leq C \sum_{m \geq 1} \sum_{j=0}^{[m / 2]}\left(\begin{array}{c}
m \\
j
\end{array}\right)\left\|\rho \partial_{x}^{j+1} v\right\|_{L^{2}}^{1 / 2}\left\|\rho \partial_{x}^{j+2} v\right\|_{L^{2}}^{1 / 2}\left\|A \rho \partial_{y} \partial_{x}^{m-j} v\right\|_{L^{2}}\left\|\rho \partial_{x}^{m} v\right\|_{L^{2}} \tau^{2 m} M_{m}^{2} \\
+C \sum_{m \geq 1} \sum_{j=[m / 2]+1}^{m}\left(\begin{array}{c}
m \\
j
\end{array}\right)\left\|\rho \partial_{x}^{j+1} v\right\|_{L^{2}}\left\|A \rho \partial_{y} \partial_{x}^{m-j} v\right\|_{L^{2}}^{1 / 2} \\
\times\left\|A \rho \partial_{y} \partial_{x}^{m-j+1} v\right\|_{L^{2}}^{1 / 2}\left\|\rho \partial_{x}^{m} v\right\|_{L^{2}} \tau^{2 m} M_{m}^{2}
\end{gathered}
$$

Using the notation (3.10)-(3.12) the above inequality yields that $\mathcal{N}_{2}$ is bounded by

$$
\begin{aligned}
& C \tau^{-1 / 2} \sum_{m \geq 1} \sum_{j=0}^{[m / 2]} Y_{j+1}^{1 / 2} Y_{j+2}^{1 / 2} Z_{m-j} Y_{m} \frac{\left(\begin{array}{c}
m \\
j
\end{array}\right) M_{m}}{M_{j+1}^{1 / 2} M_{j+2}^{1 / 2} M_{m-j}(j+1)^{1 / 2} m^{1 / 2}} \\
& \quad+C \tau^{-1 / 2} \sum_{m \geq 1} \sum_{j=[m / 2]+1}^{m} Y_{j+1} Z_{m-j}^{1 / 2} Z_{m-j+1}^{1 / 2} Y_{m} \frac{\left(\begin{array}{c}
m \\
j
\end{array}\right) M_{m}}{M_{j+1} M_{m-j}^{1 / 2} M_{m-j+1}^{1 / 2}(j+1)^{1 / 2} m^{1 / 2}}
\end{aligned}
$$

where $C=C(\alpha, r, G)$ is a sufficiently large constant. Using the explicit definition of $M_{m}$ in (3.1), we get the bound

$$
\left(\begin{array}{c}
m \\
j
\end{array}\right) \frac{M_{m}}{M_{j+1}^{1 / 2} M_{j+2}^{1 / 2} M_{m-j}(j+1)^{1 / 2} m^{1 / 2}} \leq \frac{(m+1)^{r}(j+1)^{3 / 2}}{(j+1)^{r+1 / 2}(m-j+1)^{r} m^{1 / 2}} \leq C(j+2)^{1 / 2-r}
$$


for all $0 \leq j \leq m / 2$, and

$$
\begin{aligned}
\left(\begin{array}{c}
m \\
j
\end{array}\right) \frac{M_{m}}{M_{j+1} M_{m-j}^{1 / 2} M_{m-j+1}^{1 / 2}(j+1)^{1 / 2} m^{1 / 2}} & \leq \frac{(m+1)^{r}(j+1)(m-j+1)^{1 / 2}}{(j+1)^{r}(m-j+1)^{r}(j+1)^{1 / 2} m^{1 / 2}} \\
& \leq C(m-j+1)^{1 / 2-r}
\end{aligned}
$$

for all $m / 2 \leq j \leq m$, where $C=C(r)$ is a positive constant. We have thus proven that

$$
\begin{aligned}
\mathcal{N}_{2} \leq C \tau^{-1 / 2} \sum_{m \geq 1} \sum_{j=0}^{[m / 2]} Y_{j+1}^{1 / 2} Y_{j+2}^{1 / 2} Z_{m-j} Y_{m}(j+1)^{1 / 2-r} \\
+C \tau^{-1 / 2} \sum_{m \geq 1} \sum_{j=[m / 2]+1}^{m} Y_{j+1} Z_{m-j}^{1 / 2} Z_{m-j+1}^{1 / 2} Y_{m}(m-j+1)^{1 / 2-r}
\end{aligned}
$$

and so, by the discrete Hölder and Young inequalities (cf. (4.14)), we obtain

$$
\mathcal{N}_{2} \leq C \tau^{-1 / 2}\|v\|_{Z_{\tau}}\|v\|_{Y_{\tau}}^{2}
$$

as long as $r>1$, for some positive constant $C=C\left(\tau_{0}, \alpha, r, \tau_{E}, G\right)>0$.

4.4. $\mathcal{N}_{3}$ bound. It is left to bound $\mathcal{N}_{3}$. In this term a slight complication arises since we need to take higher derivatives of $\partial_{x} a$. In order to deal with this we use (3.18), and recall that $\tau \leq \tau_{0} \ll \tau_{*}<\tau_{E}$. From the Leibniz rule, (3.18), and (4.5), we get

$$
\begin{gathered}
\mathcal{N}_{3} \leq \sum_{m \geq 1} \sum_{j=0}^{m} \sum_{k=0}^{j}\left(\begin{array}{c}
m \\
j
\end{array}\right)\left(\begin{array}{l}
j \\
k
\end{array}\right)\left\|\partial_{x}^{k+1} a\right\|_{L^{\infty}}\left\|\partial_{x}^{j-k} W(v) \rho \partial_{y} \partial_{x}^{m-j} v\right\|_{L^{2}}\left\|\rho \partial_{x}^{m} v\right\|_{L^{2}} \tau^{2 m} M_{m}^{2} \\
\leq C \sum_{m \geq 1} \sum_{j=0}^{m} \sum_{k=0}^{j} \frac{m !}{(m-j) !(j-k) !(k+1)^{r}}\left\|A^{-1}\right\|_{L^{\infty}}\left\|\partial_{x}^{j-k} W(v)\right\|_{L^{\infty}} \\
\leq C \sum_{m \geq 1} \sum_{j=0}^{m} \sum_{k=0}^{j} \frac{\times A \rho \partial_{y} \partial_{x}^{m-j} v\left\|_{L^{2}}\right\| \rho \partial_{x}^{m} v \|_{L^{2}} \tau^{2 m-k-1} M_{m}^{2}}{(m-j) !(j-k) !(k+1)^{r}}\left\|\rho \partial_{x}^{j-k} v\right\|_{L^{2}}^{1 / 2}\left\|\rho \partial_{x}^{j-k+1} v\right\|_{L^{2}}^{1 / 2} \\
\times\left\|A \rho \partial_{y} \partial_{x}^{m-j} v\right\|_{L^{2}}\left\|\rho \partial_{x}^{m} v\right\|_{L^{2}} \tau^{2 m-k-1} M_{m}^{2}
\end{gathered}
$$

for some constant $C=C\left(G, \tau_{E}, \tau_{0}\right)>0$ that depends on the analyticity radius and the analytic norm of the underlying Euler flow. Using the notation introduced in (3.10)-(3.12), the above estimate becomes

$$
\mathcal{N}_{3} \leq C \tau^{-3 / 4} \sum_{m \geq 1} \sum_{j=0}^{m} \sum_{k=0}^{j} X_{j-k}^{1 / 2} Y_{j-k+1}^{1 / 2} Z_{m-j} Y_{m} \Gamma_{m, j, k}
$$

for some positive constant $C=C\left(G, \tau_{E}, \tau_{0}\right)$, where

$$
\Gamma_{m, j, k}=\frac{m !}{(m-j) !(j-k) !} \cdot \frac{M_{m}}{(k+1)^{r} M_{j-k}^{1 / 2} M_{j-k+1}^{1 / 2}(j-k+1)^{1 / 4} M_{m-j} m^{1 / 2}} .
$$


Using the explicit definition of $M_{m}$, we obtain that

$$
\begin{aligned}
\Gamma_{m, j, k} & \leq C \frac{(m+1)^{r}(j-k+1)^{1 / 4}}{(k+1)^{r}(j-k+1)^{r}(m-j+1)^{r} m^{1 / 2}} \\
& \leq C \frac{(m+1)^{r}}{(k+1)^{r}(j-k+1)^{r}(m-j+1)^{r} m^{1 / 4}} \\
& \leq C \begin{cases}(k+1)^{-r-1 / 4}, & j \leq m / 2 \\
(k+1)^{-r-1 / 4}, & j \geq m / 2, k \leq j / 2 \\
(m-j+1)^{-r-1 / 4}, & j \geq m / 2, k \geq j / 2,\end{cases}
\end{aligned}
$$

for some positive constant $C=C(r)$. If $r>1$ it then follows from (4.24), (4.26), and the discrete Hölder and Young inequalities that

$$
\mathcal{N}_{3} \leq C \tau^{-3 / 4}\|v\|_{X_{\tau}}^{1 / 2}\|v\|_{Z_{\tau}}\|v\|_{Y_{\tau}}^{3 / 2}
$$

for some positive constant $C$. Lastly, combining (4.6), (4.15), (4.22), and (4.27) we obtain

$$
\begin{aligned}
\mathcal{N} & \leq C \tau^{-1}\|v\|_{Z_{\tau}}\left(\|v\|_{X_{\tau}}\|v\|_{Y_{\tau}}+\|v\|_{X_{\tau}}^{3 / 2}\|v\|_{Y_{\tau}}^{1 / 2}+\|v\|_{Y_{\tau}}^{2}+\|v\|_{X_{\tau}}^{1 / 2}\|v\|_{Y_{\tau}}^{3 / 2}\right) \\
& \leq C \tau^{-1}\|v\|_{Z_{\tau}}\left(\|v\|_{X_{\tau}}^{2}+\|v\|_{Y_{\tau}}^{2}\right)
\end{aligned}
$$

for some positive constant $C$ depending on $r>1$, the underlying Euler flow, and $\tau_{0}$. This concludes the bounds on the nonlinear term.

\section{The bound of the linear term}

In this section we bound

$$
\mathcal{L}=\sum_{m \geq 0}\left\|\rho \partial_{x}^{m} L(v)\right\|_{L^{2}}\left\|\rho \partial_{x}^{m} v\right\|_{L^{2}} \tau^{2 m} M_{m}^{2} \leq \mathcal{L}_{0}+\mathcal{L}_{1}+\mathcal{L}_{2}+\mathcal{L}_{3}+\mathcal{L}_{4}+\mathcal{L}_{5}+\mathcal{L}_{6}
$$

where by (2.9) we have

$$
\begin{aligned}
L(v)= & \partial_{x} W(v) \partial_{y} \phi U+\partial_{x} v(1-\phi) U+\partial_{y} v \Phi \partial_{x} U \\
& \quad-\partial_{y} v \Phi \partial_{x} a U-W(v) \partial_{y} \phi \partial_{x} a U+v(1-\phi) \partial_{x} U \\
= & L_{1}+L_{2}+L_{3}+L_{4}+L_{5}+L_{6},
\end{aligned}
$$

and where we denoted

$$
\mathcal{L}_{i}=\sum_{m \geq 1}\left\|\rho \partial_{x}^{m} L_{i}\right\|_{L^{2}}\left\|\rho \partial_{x}^{m} v\right\|_{L^{2}} \tau^{2 m} M_{m}^{2}
$$

for each $i \in\{1, \ldots, 6\}$, and

$$
\mathcal{L}_{0}=\|\rho L(v)\|_{L^{2}}\|\rho v\|_{L^{2}} .
$$

From (3.16)-(3.18) and the Leibniz rule, we may prove that the functions $U(\cdot, t)$, $\partial_{x} U(\cdot, t)$, and $\partial_{x} a(\cdot, t) U(\cdot, t)$ have radius of analyticity at least $\tau_{*}>0$ on $[0, T]$, i.e., upon possibly increasing $G$ we have

$$
\left\|\partial_{x}^{k} U\right\|_{L^{\infty}\left([0, T] ; L^{\infty}\right)}+\left\|\partial_{x}^{k}\left(\partial_{x} U\right)\right\|_{L^{\infty}\left([0, T] ; L^{\infty}\right)}+\left\|\partial_{x}^{k}\left(\partial_{x} a U\right)\right\|_{L^{\infty}\left([0, T] ; L^{\infty}\right)} \leq \frac{G}{\tau_{*}^{k} M_{k}}
$$


for all $k \geq 0$. Recalling from (3.21) that $\tau_{*}$ was chosen to satisfy $\tau_{*} /\left(1+C_{0} G\right) \geq \tau_{0} \geq \tau$, we may write

$$
\frac{\tau(t)}{\tau_{*}} \leq \frac{1}{2}
$$

for all $t \in[0, T]$. Also, note that we have $\left|\partial_{y} \phi(y)\right| \leq C /\langle y\rangle^{\theta+1}$, and since $\rho(y)=\langle y\rangle^{\alpha}$, we have $\rho \partial_{y} \phi \in L_{y}^{2}([0, \infty))$ if and only if $\theta>\alpha-1 / 2$. Lastly, $\Phi \in L_{y}^{\infty}([0, \infty))$ whenever $\theta>1$.

5.1. $\mathcal{L}_{0}$ bound. Using the Hölder inequality and Lemma 4.2, we have

$$
\begin{aligned}
& \mathcal{L}_{0} \leq C\|\rho v\|_{L^{2}}\left(\left\|\partial_{x} W(v)\right\|_{L_{y}^{\infty} L_{x}^{2}}\left\|\rho \partial_{y} \phi\right\|_{L_{y}^{2}}\|U\|_{L_{x}^{\infty}}+\left\|\rho \partial_{x} v\right\|_{L^{2}}\|1-\phi\|_{L_{y}^{\infty}}\|U\|_{L_{x}^{\infty}}\right. \\
&+\left\|\rho A \partial_{y} v\right\|_{L^{2}}\left\|A^{-1}\right\|_{L_{x}^{\infty}}\|\Phi\|_{L_{y}^{\infty}}\left\|\partial_{x} U\right\|_{L_{x}^{\infty}} \\
&+\left\|\rho A \partial_{y} v\right\|_{L^{2}}\left\|A^{-1}\right\|_{L_{x}^{\infty}}\|\Phi\|_{L_{y}^{\infty}}\left\|\partial_{x} a U\right\|_{L_{x}^{\infty}} \\
&\left.+\|W(v)\|_{L_{y}^{\infty} L_{x}^{2}}\left\|\rho \partial_{y} \phi\right\|_{L_{y}^{2}}\left\|\partial_{x} a U\right\|_{L_{x}^{\infty}}+\|\rho v\|_{L^{2}}\|1-\phi\|_{L_{y}^{\infty}}\left\|\partial_{x} U\right\|_{L_{x}^{\infty}}\right) \\
& \leq C \tau^{-1} X_{0}\left(X_{1}+Z_{0}+X_{0}\right) \\
& \leq C \tau^{-1}\|v\|_{X_{\tau}}\left(\|v\|_{X_{\tau}}+\|v\|_{Z_{\tau}}\right)
\end{aligned}
$$

for some positive constant $C=C\left(r, G, \tau_{*}\right)$. Here we used $\theta>\alpha-1 / 2>0$, so that $1-$ $\phi, \Phi \in L_{y}^{\infty}$ and $\rho \partial_{y} \phi \in L_{y}^{2}$.

5.2. $\mathcal{L}_{1}$ bound. In order to bound

$$
\mathcal{L}_{1}=\sum_{m \geq 1}\left\|\rho \partial_{x}^{m}\left(\partial_{x} W(v) \partial_{y} \phi U\right)\right\|_{L^{2}}\left\|\rho \partial_{x}^{m} v\right\|_{L^{2}} \tau^{2 m} M_{m}^{2},
$$

we use the Leibniz rule and the Hölder inequality to obtain

$$
\mathcal{L}_{1} \leq \sum_{m \geq 1} \sum_{j=0}^{m}\left(\begin{array}{c}
m \\
j
\end{array}\right)\left\|\partial_{x}^{j+1} W(v)\right\|_{L_{y}^{\infty} L_{x}^{2}}\left\|\rho \partial_{y} \phi\right\|_{L_{y}^{2}}\left\|\partial_{x}^{m-j} U\right\|_{L_{x}^{\infty}}\left\|\rho \partial_{x}^{m} v\right\|_{L^{2}} \tau^{2 m} M_{m}^{2} .
$$

From Lemma 4.2 and (5.5), after recalling the notation (3.10)-(3.12), we obtain

$$
\begin{aligned}
\mathcal{L}_{1} & \leq C G \sum_{m \geq 1} \sum_{j=0}^{m}\left(\begin{array}{c}
m \\
j
\end{array}\right)\left\|\rho \partial_{x}^{j+1} v\right\|_{L^{2}} \frac{1}{\tau_{*}^{m-j} M_{m-j}}\left\|\rho \partial_{x}^{m} v\right\|_{L^{2}} \tau^{2 m} M_{m}^{2} \\
& \leq C G \sum_{m \geq 1} \sum_{j=0}^{m} Y_{j+1} Y_{m}\left(\frac{\tau}{\tau_{*}}\right)^{m-j}\left(\left(\begin{array}{c}
m \\
j
\end{array}\right) \frac{M_{m}^{2}}{M_{j+1}(j+1)^{1 / 2} M_{m} m^{1 / 2} M_{m-j}}\right) .
\end{aligned}
$$

Using the definition of $M_{m}$ we may bound

$$
\left(\begin{array}{c}
m \\
j
\end{array}\right) \frac{M_{m}^{2}}{M_{j+1}(j+1)^{1 / 2} M_{m} m^{1 / 2} M_{m-j}} \leq \frac{m ! M_{m}}{j ! M_{j}(m-j) ! M_{m-j}} \frac{j}{(j+1)^{1 / 2} m^{1 / 2}} \leq C
$$

for some $C=C(r)>0$, for all $0 \leq j \leq m$. Since by (5.6) we have $\tau / \tau_{*} \leq 1 / 2,(5.8)$ and the above estimate and give

$$
\mathcal{L}_{1} \leq C \sum_{m \geq 1} \sum_{j=0}^{m} Y_{j+1} Y_{m} \frac{1}{2^{m-j}} \leq C\|u\|_{Y_{\tau}}^{2}
$$

for some constant $C=C(G, r)>0$, by using the discrete Young and Hölder inequalities. 
5.3. $\mathcal{L}_{2}$ bound. From the Leibniz rule and the Hölder inequality we have

$$
\begin{aligned}
\mathcal{L}_{2} & =\sum_{m \geq 1}\left\|\rho \partial_{x}^{m}\left(\partial_{x} v(1-\phi) U\right)\right\|_{L^{2}}\left\|\rho \partial_{x}^{m} v\right\|_{L^{2}} \tau^{2 m} M_{m}^{2} \\
& \leq \sum_{m \geq 1} \sum_{j=0}^{m}\left(\begin{array}{c}
m \\
j
\end{array}\right)\left\|\rho \partial_{x}^{j+1} v\right\|_{L^{2}}\|1-\phi\|_{L_{y}^{\infty}}\left\|\partial_{x}^{m-j} U\right\|_{L_{x}^{\infty}}\left\|\rho \partial_{x}^{m} v\right\|_{L^{2}} \tau^{2 m} M_{m}^{2} .
\end{aligned}
$$

From (5.5) and the above estimate we obtain

$$
\begin{aligned}
\mathcal{L}_{2} & \leq C G \sum_{m \geq 1} \sum_{j=0}^{m}\left(\begin{array}{c}
m \\
j
\end{array}\right)\left\|\rho \partial_{x}^{j+1} v\right\|_{L^{2}} \frac{1}{\tau_{*}^{m-j} M_{m-j}}\left\|\rho \partial_{x}^{m} v\right\|_{L^{2}} \tau^{2 m} M_{m}^{2} \\
& \leq C G \sum_{m \geq 1} \sum_{j=0}^{m} Y_{j+1} Y_{m}\left(\frac{\tau}{\tau_{*}}\right)^{m-j}\left(\left(\begin{array}{c}
m \\
j
\end{array}\right) \frac{M_{m}^{2}}{M_{j+1}(j+1)^{1 / 2} M_{m} m^{1 / 2} M_{m-j}}\right)
\end{aligned}
$$

which bounds $\mathcal{L}_{2}$ from above exactly by the right side of (5.8), and we obtain

$$
\mathcal{L}_{2} \leq C\|u\|_{Y_{\tau}}^{2}
$$

for some constant $C=C(G, r)>0$.

5.4. $\mathcal{L}_{3}$ bound. As above, from the Leibniz rule and the Hölder inequality we obtain

$$
\begin{aligned}
\mathcal{L}_{3} & =\sum_{m \geq 1}\left\|\rho \partial_{x}^{m}\left(\partial_{y} v \Phi \partial_{x} U\right)\right\|_{L^{2}}\left\|\rho \partial_{x}^{m} v\right\|_{L^{2}} \tau^{2 m} M_{m}^{2} \\
& \leq \sum_{m \geq 1} \sum_{j=0}^{m}\left(\begin{array}{c}
m \\
j
\end{array}\right)\left\|\rho \partial_{y} \partial_{x}^{j} v \Phi \partial_{x}^{m-j+1} U\right\|_{L^{2}}\left\|\rho \partial_{x}^{m} v\right\|_{L^{2}} \tau^{2 m} M_{m}^{2} \\
& \leq 2 \sum_{m \geq 1} \sum_{j=0}^{m}\left(\begin{array}{c}
m \\
j
\end{array}\right)\left\|A \rho \partial_{y} \partial_{x}^{j} v\right\|_{L^{2}}\|\Phi\|_{L_{y}^{\infty}}\left\|\partial_{x}^{m-j}\left(\partial_{x} U\right)\right\|_{L_{x}^{\infty}}\left\|\rho \partial_{x}^{m} v\right\|_{L^{2}} \tau^{2 m} M_{m}^{2}
\end{aligned}
$$

since $\|1 / A\|_{L_{x}^{\infty}} \leq 2$ on $[0, T]$. Inserting estimate (5.5) into the bound (5.14) above, we get

$$
\begin{aligned}
\mathcal{L}_{3} & \leq C G \sum_{m \geq 1} \sum_{j=0}^{m}\left(\begin{array}{c}
m \\
j
\end{array}\right)\left\|A \rho \partial_{y} \partial_{x}^{j} v\right\|_{L^{2}} \frac{1}{\tau_{*}^{m-j} M_{m-j}}\left\|\rho \partial_{x}^{m} v\right\|_{L^{2}} \tau^{2 m} M_{m}^{2} \\
& \leq C G \sum_{m \geq 1} \sum_{j=0}^{m} Z_{j} X_{m}\left(\frac{\tau}{\tau_{*}}\right)^{m-j}\left(\left(\begin{array}{c}
m \\
j
\end{array}\right) \frac{M_{m}}{M_{j} M_{m-j}}\right) .
\end{aligned}
$$

Recall that we have $m ! M_{m} /\left(j ! M_{j}(m-j) ! M_{m-j}\right) \leq C$ by (3.19), and therefore the estimate (5.15) combined with the discrete Young and Hölder inequalities gives

$$
\mathcal{L}_{3} \leq C \sum_{m \geq 1} \sum_{j=0}^{m} Z_{j} X_{m} \frac{1}{2^{m-j}} \leq C\|v\|_{X_{\tau}}\|v\|_{Z_{\tau}}
$$

for some positive constant $C=C(r, G)$. 
5.5. $\mathcal{L}_{4}$ bound. Similarly to (5.14) we obtain

$$
\mathcal{L}_{4} \leq 2 \sum_{m \geq 1} \sum_{j=0}^{m}\left(\begin{array}{c}
m \\
j
\end{array}\right)\left\|A \rho \partial_{y} \partial_{x}^{j} v\right\|_{L^{2}}\|\Phi\|_{L_{y}^{\infty}}\left\|\partial_{x}^{m-j}\left(\partial_{x} a U\right)\right\|_{L_{x}^{\infty}}\left\|\rho \partial_{x}^{m} v\right\|_{L^{2}} \tau^{2 m} M_{m}^{2}
$$

Using (5.5) to bound $\left\|\partial_{x}^{m-j}\left(\partial_{x} a U\right)\right\|_{L_{x}^{\infty}}$, and (3.19) to treat the combinatorial remainder, we conclude that

$$
\mathcal{L}_{4} \leq C\|v\|_{X_{\tau}}\|v\|_{Z_{\tau}}
$$

for some positive constant $C=C(r, G)$.

5.6. $\mathcal{L}_{5}$ bound. In order to bound $\mathcal{L}_{5}$ we use the Leibniz rule and Hölder inequalities and obtain

$$
\begin{aligned}
\mathcal{L}_{5} & =\sum_{m \geq 1}\left\|\rho \partial_{x}^{m}\left(W(v) \partial_{y} \phi \partial_{x} a U\right)\right\|_{L^{2}}\left\|\rho \partial_{x}^{m} v\right\|_{L^{2}} \tau^{2 m} M_{m}^{2} \\
& \leq \sum_{m \geq 1} \sum_{j=0}^{m}\left(\begin{array}{c}
m \\
j
\end{array}\right)\left\|\partial_{x}^{j} W(v)\right\|_{L_{y}^{\infty} L_{x}^{2}}\left\|\rho \partial_{y} \phi\right\|_{L_{y}^{2}}\left\|\partial_{x}^{m-j}\left(\partial_{x} a U\right)\right\|_{L_{x}^{\infty}}\left\|\rho \partial_{x}^{m} v\right\|_{L^{2}} \tau^{2 m} M_{m}^{2}
\end{aligned}
$$

Using Lemma 4.2 and (5.5), estimate (5.19) implies

$$
\begin{aligned}
\mathcal{L}_{5} & \leq C G \sum_{m \geq 1} \sum_{j=0}^{m}\left(\begin{array}{c}
m \\
j
\end{array}\right)\left\|\rho \partial_{x}^{j} v\right\|_{L^{2}} \frac{1}{\tau_{*}^{m-j} M_{m-j}}\left\|\rho \partial_{x}^{m} v\right\|_{L^{2}} \tau^{2 m} M_{m}^{2} \\
& \leq C G \sum_{m \geq 1} \sum_{j=0}^{m} X_{j} X_{m}\left(\frac{\tau}{\tau_{*}}\right)^{m-j}\left(\left(\begin{array}{c}
m \\
j
\end{array}\right) \frac{M_{m}}{M_{j} M_{m-j}}\right) .
\end{aligned}
$$

From (3.19), (5.6), the discrete Young and Hölder inequalities, and the above estimate, we have

$$
\mathcal{L}_{5} \leq C \sum_{m \geq 1} \sum_{j=0}^{m} X_{j} X_{m} \frac{1}{2^{m-j}} \leq C\|v\|_{X_{\tau}}^{2}
$$

for some positive constant $C=C(r, G)$.

5.7. $\mathcal{L}_{6}$ bound. As in (5.19), we have the bound

$$
\begin{aligned}
\mathcal{L}_{6} & =\sum_{m \geq 1}\left\|\rho \partial_{x}^{m}\left(v(1-\phi) \partial_{x} U\right)\right\|_{L^{2}}\left\|\rho \partial_{x}^{m} v\right\|_{L^{2}} \tau^{2 m} M_{m}^{2} \\
& \leq \sum_{m \geq 1} \sum_{j=0}^{m}\left(\begin{array}{c}
m \\
j
\end{array}\right)\left\|\rho \partial_{x}^{j} v\right\|_{L^{2}}\|1-\phi\|_{L_{y}^{\infty}}\left\|\partial_{x}^{m-j}\left(\partial_{x} U\right)\right\|_{L_{x}^{\infty}}\left\|\rho \partial_{x}^{m} v\right\|_{L^{2}} \tau^{2 m} M_{m}^{2}
\end{aligned}
$$

From (3.19), (5.5), and (5.6), similarly to (5.21) we obtain

$$
\mathcal{L}_{6} \leq C\|v\|_{X_{\tau}}^{2}
$$

for some positive constant $C=C(r, G)$.

We summarize the bounds on the linear term $\mathcal{L}$ by collecting the upper bounds of (5.7), (5.10), (5.13), (5.16), (5.18), (5.21), and (5.23), as

$$
\mathcal{L} \leq C \tau^{-1}\|v\|_{X_{\tau}}\left(\|v\|_{X_{\tau}}+\|v\|_{Z_{\tau}}\right)+C\|v\|_{Y_{\tau}}^{2}
$$

for some sufficiently large positive constant $C$ depending on $r$, the analyticity radius, and the analytic norm of the underlying Euler flow. 


\section{The bound on the force term}

In this section we bound

$$
\mathcal{F}=\sum_{m \geq 0}\left|\left\langle\rho \partial_{x}^{m} F, \rho \partial_{x}^{m} v\right\rangle\right| \tau^{2 m} M_{m}^{2} \leq \mathcal{F}_{1}+\mathcal{F}_{2}+\mathcal{F}_{3}+\mathcal{F}_{4},
$$

where by (2.10) we have

$$
F=\left(\phi(1-\phi)+\Phi \partial_{y} \phi\right) U \partial_{x} U-\partial_{y} \phi \Phi \partial_{x} a U^{2}-\partial_{y y} \phi A^{2} U-\phi \partial_{x} P=F_{1}+F_{2}+F_{3}+F_{4},
$$

and we have denoted

$$
\mathcal{F}_{i}=\sum_{m \geq 0}\left\|\rho \partial_{x}^{m} F_{i}\right\|_{L^{2}}\left\|\rho \partial_{x}^{m} v\right\|_{L^{2}} \tau^{2 m} M_{m}^{2}
$$

for all $i \in\{1, \ldots, 4\}$. To simplify the analysis, as in (5.5), from (3.16)-(3.18) and the Leibniz rule we may assume that the functions $U(\cdot, t) \partial_{x} U(\cdot, t), \partial_{x} a(\cdot, t) U^{2}(\cdot, t)$, $A^{2}(\cdot, t) U(\cdot, t)$, and $\partial_{x} P(\cdot, t)$ are real-analytic for all $t \in[0, T]$, with uniform analyticity radius bounded from below by $\tau_{*}$. That is, by possibly increasing $G$ we may assume that

$$
\left\|\partial_{x}^{j}\left(U \partial_{x} U\right)\right\|_{L_{x}^{2}}+\left\|\partial_{x}^{j}\left(\partial_{x} a U^{2}\right)\right\|_{L_{x}^{2}}+\left\|\partial_{x}^{j}\left(A^{2} U\right)\right\|_{L_{x}^{2}}+\left\|\partial_{x}^{j}\left(\partial_{x} P\right)\right\|_{L_{x}^{2}} \leq \frac{G}{\tau_{*}^{j} M_{j}}
$$

for all $j \geq 0$ and all $t \in[0, T]$. Here $M_{j}$ is as defined in (3.1), and for all $t \in[0, T]$ we additionally have

$$
\frac{\tau(t)}{\tau_{*}} \leq \frac{1}{2}
$$

6.1. $\mathcal{F}_{1}$ bound. From the definition of $\mathcal{F}_{1}($ cf. $(6.1))$ and the Hölder inequality we have

$$
\mathcal{F}_{1} \leq \sum_{m \geq 0}\left(\|\rho \phi(1-\phi)\|_{L_{y}^{2}}+\left\|\rho \Phi \partial_{y} \phi\right\|_{L_{y}^{2}}\right)\left\|\partial_{x}^{m}\left(U \partial_{x} U\right)\right\|_{L_{x}^{2}}\left\|\rho \partial_{x}^{m} v\right\|_{L^{2}} \tau^{2 m} M_{m}^{2} .
$$

In order to bound the $L_{y}^{2}$-terms we first note that

$$
\|\rho \phi(1-\phi)\|_{L_{y}^{2}} \leq\|\rho \phi\|_{L_{y}^{2}}\|1-\phi\|_{L_{y}^{\infty}} \leq C(\alpha, \theta)
$$

as long as $\rho(y) \phi(y)=1 /\langle y\rangle^{\theta-\alpha} \in L_{y}^{2}$, i.e., if we impose that $\theta>\alpha+1 / 2$. Similarly, as long as $\theta>\alpha-1 / 2$ we have $\rho \partial_{y} \phi \in L_{y}^{2}$, and if additionally $\theta>1$, we have $\Phi \in L_{y}^{\infty}$, so that

$$
\left\|\rho \Phi \partial_{y} \phi\right\|_{L_{y}^{2}} \leq\left\|\rho \partial_{y} \phi\right\|_{L_{y}^{2}}\|\Phi\|_{L_{y}^{\infty}} \leq C(\alpha, \theta) .
$$

Combining (6.2)-(6.6) we obtain

$$
\mathcal{F}_{1} \leq C G \sum_{m \geq 0} \frac{1}{\tau_{*}^{m} M_{m}}\left\|\rho \partial_{x}^{m} v\right\|_{L^{2}} \tau^{2 m} M_{m}^{2} \leq C G \sum_{m \geq 0}\left(\frac{\tau}{\tau_{*}}\right)^{m} X_{m} \leq C G \sum_{m \geq 0} \frac{1}{2^{m}} X_{m}
$$

so that from the discrete Cauchy-Schwartz inequality we have

$$
\mathcal{F}_{1} \leq C\|v\|_{X_{\tau}}
$$

for some sufficiently large constant $C=C(G, \alpha, \theta)>0$. 
6.2. $\mathcal{F}_{2}$ bound. We obtain from the Hölder inequality that

$$
\begin{aligned}
\mathcal{F}_{2} & =\sum_{m \geq 0}\left\|\rho \partial_{x}^{m}\left(\partial_{y} \phi \Phi \partial_{x} a U^{2}\right)\right\|_{L^{2}}\left\|\rho \partial_{x}^{m} v\right\|_{L^{2}} \tau^{2 m} M_{m}^{2} \\
& \leq \sum_{m \geq 0}\left\|\rho \partial_{y} \phi\right\|_{L_{y}^{2}}\|\Phi\|_{L_{y}^{\infty}}\left\|\partial_{x}^{m}\left(\partial_{x} a U^{2}\right)\right\|_{L_{x}^{2}}\left\|\rho \partial_{x}^{m} v\right\|_{L^{2}} \tau^{2 m} M_{m}^{2} .
\end{aligned}
$$

Assuming that $\theta>\alpha-1 / 2$ and $\theta>1$, we have

$$
\left\|\rho \partial_{y} \phi\right\|_{L_{y}^{2}}\|\Phi\|_{L_{y}^{\infty}} \leq C(\alpha, \theta)
$$

and therefore, by using (6.2)-(6.3) we obtain (similarly to (6.7)) that

$$
\mathcal{F}_{2} \leq C G \sum_{m \geq 0} \frac{1}{\tau_{*}^{m} M_{m}}\left\|\rho \partial_{x}^{m} v\right\|_{L^{2}} \tau^{2 m} M_{m}^{2} \leq C G \sum_{m \geq 0} \frac{1}{2^{m}} X_{m} .
$$

Thus we obtain by the Cauchy-Schwarz inequality that

$$
\mathcal{F}_{2} \leq C\|v\|_{X_{\tau}}
$$

for some sufficiently large constant $C=C(G, \alpha, \theta)>0$.

6.3. $\mathcal{F}_{3}$ bound. In order to bound $\mathcal{F}_{3}$ we observe that

$$
\rho(y) \partial_{y y} \phi(y)=\langle y\rangle^{\alpha}\left(\theta(2+\theta) \frac{y^{2}}{\langle y\rangle^{4+\theta}}-\theta \frac{1}{\langle y\rangle^{2+\theta}}\right)
$$

so that

$$
\left|\rho(y) \partial_{y y} \phi(y)\right| \leq \theta(2+\theta) \frac{1}{\langle y\rangle^{2+\theta-\alpha}}
$$

for all $y \geq 0$. Therefore, if $\theta>\alpha-3 / 2$ we have that $\rho \partial_{y y} \phi \in L_{y}^{2}([0, \infty))$, and hence similarly to $(6.8)$ we obtain

$$
\mathcal{F}_{3} \leq \sum_{m \geq 0}\left\|\rho \partial_{y y} \phi\right\|_{L_{y}^{2}}\left\|\partial_{x}^{m}\left(A^{2} U\right)\right\|_{L_{x}^{2}}\left\|\rho \partial_{x}^{m} v\right\|_{L^{2}} \tau^{2 m} M_{m}^{2} \leq C\|v\|_{X_{\tau}}
$$

for some positive constant $C=C(G, \alpha, \theta)$. In the above estimate we also used (6.2) and (6.3).

6.4. $\mathcal{F}_{4}$ bound. Similarly to $(6.8)$, it follows from $\rho \phi \in L_{y}^{2}([0, \infty))$ whenever $\theta>\alpha+1 / 2,(6.2)$, and (6.3) that

$$
\mathcal{F}_{4} \leq C\|v\|_{X_{\tau}}
$$

for some positive constant $C=C(G, \alpha, \theta)$.

Combining the estimates $(6.8),(6.11),(6.12)$, and (6.13) we obtain that also

$$
\mathcal{F} \leq C\|v\|_{X_{\tau}}
$$

for a suitable constant $C>0$ depending on the underlying Euler flow, $\alpha$, and $\theta$.

Appendix A. Details regarding the change of variables. Here we provide details on how the Prandtl equations (1.1)-(1.8) become the system (2.6)-(2.10), 
under the change of variables (2.4)-(2.5). First, in order to use the assumption $u(x, Y)-U(x) \rightarrow 0$ as $Y \rightarrow \infty$ for every $x \in \mathbb{R}$, we substitute

$$
\begin{aligned}
& \tilde{u}(x, Y, t)=u(x, Y, t)-U(x, t), \\
& \tilde{w}(x, Y, t)=-\partial_{x} \int_{0}^{Y} \tilde{u}(x, \zeta, t) d \zeta .
\end{aligned}
$$

From the incompressibility condition (1.2), the boundary condition (1.5), and the substitutions (A.1)-(A.2) we obtain

$$
w(x, Y)=-\partial_{x} \int_{0}^{Y} u(x, \zeta) d \zeta=-Y \partial_{x} U+\tilde{w}(x, Y) .
$$

Therefore, (1.1) now reads

$$
\partial_{t} \tilde{u}-Y \partial_{x} U \partial_{Y} \tilde{u}-\partial_{Y Y} \tilde{u}+\tilde{u} \partial_{x} \tilde{u}+\tilde{w} \partial_{Y} \tilde{u}+\left(U \partial_{x} \tilde{u}+\tilde{u} \partial_{x} U\right)=-\partial_{t} U-U \partial_{x} U-\partial_{x} P=0,
$$

since $(U, P)$ is the trace of a solution to the Euler equations. The boundary conditions for $\tilde{u}$ are

$$
\begin{aligned}
& \left.\tilde{u}(x, Y, t)\right|_{Y=0}=-U(x, t), \\
& \lim _{Y \rightarrow \infty} \tilde{u}(x, Y, t)=0 .
\end{aligned}
$$

The formulation (A.3)-(A.5) was successfully used in [4, 23, 31, 32] to obtain a local in time analytic solution of the Prandtl equations which vanishes exponentially as $Y \rightarrow \infty$. The need for this exponential decay is due to the term $Y \partial_{x} U \partial_{Y} \tilde{u}$ on the left side of (A.3). In order to obtain solutions which decay only algebraically for large $Y$ we introduce the second change of variables

$$
\begin{aligned}
y & =Y A(x, t), \\
\bar{v}(x, y, t) & =\tilde{u}(x, Y, t), \\
\bar{w}(x, y, t) & =\tilde{w}(x, Y, t),
\end{aligned}
$$

where $A(x, t)>0$ is a real-analytic function on $\mathbb{R} \times[0, T]$, to be determined (cf. (A.19)(A.20) below). Note that the change of variables (A.6)-(A.8) does not change the boundary conditions as we still have (we used here that $A(x, t)>0$ )

$$
\begin{aligned}
& \left.\bar{v}(x, y, t)\right|_{y=0}=-U(x, t), \\
& \lim _{y \rightarrow \infty} \bar{v}(x, y, t)=0,
\end{aligned}
$$

for all $x \in \mathbb{R}$ and $t \geq 0$. By the chain rule, from (A.6)-(A.7) we obtain

$$
\begin{aligned}
\partial_{t} \tilde{u} & =\partial_{t} \bar{v}+\frac{y \partial_{t} A}{A} \partial_{y} \bar{v} \\
\partial_{Y} \tilde{u} & =A \partial_{y} \bar{v} \\
\partial_{Y Y} \tilde{u} & =A^{2} \partial_{y y} \bar{v} \\
\partial_{x} \tilde{u} & =\partial_{x} \bar{v}+\frac{y \partial_{x} A}{A} \partial_{y} \bar{v}
\end{aligned}
$$


The incompressibility condition $\partial_{x} \tilde{u}+\partial_{Y} \tilde{w}=0$ now reads

$$
\partial_{x} \bar{v}+\frac{y \partial_{x} A}{A} \partial_{y} \bar{v}+A \partial_{y} \bar{w}=0
$$

Using that $\bar{w}(x, 0, t)=\tilde{w}(x, 0, t)=0$, the above relation implies

$$
\bar{w}(x, y, t)=-\frac{1}{A(x, t)} \partial_{x} \int_{0}^{y} \bar{v}(x, \zeta, t) d \zeta-\frac{y \partial_{x} A(x, t)}{A^{2}(x, t)} \bar{v}(x, y, t)+\frac{\partial_{x} A(x, t)}{A^{2}(x, t)} \int_{0}^{y} \bar{v}(x, \zeta, t) d \zeta
$$

and, after denoting

$$
W(\bar{v})(x, y, t)=\int_{0}^{y} \bar{v}(x, \zeta, t) d \zeta
$$

the definition of $\bar{w}$ becomes

$$
\bar{w}=-\frac{1}{A} \partial_{x} W(\bar{v})-\frac{y \partial_{x} A}{A^{2}} \bar{v}+\frac{\partial_{x} A}{A^{2}} W(\bar{v}) .
$$

Combining the identities (A.11)-(A.14) with (A.3) and (A.16), we obtain the evolution equation for $\bar{v}$ :

$$
\begin{aligned}
\partial_{t} \bar{v}+ & \frac{y \partial_{t} A}{A} \partial_{y} \bar{v}-A^{2} \partial_{y y} \bar{v}-y \partial_{x} U \partial_{y} \bar{v}+\bar{v}\left(\partial_{x} \bar{v}+\frac{y \partial_{x} A}{A} \partial_{y} \bar{v}\right) \\
& -\left(\partial_{x} W(\bar{v})+\frac{y \partial_{x} A}{A} \bar{v}-\frac{\partial_{x} A}{A} W(\bar{v})\right) \partial_{y} \bar{v}+U \partial_{x} \bar{v}+\frac{y \partial_{x} A}{A} U \partial_{y} \bar{v}+\bar{v} \partial_{x} U=0
\end{aligned}
$$

which may be rewritten as

$$
\begin{aligned}
\partial_{t} \bar{v}- & A^{2} \partial_{y y} \bar{v}+y\left(\frac{\partial_{t} A}{A}-\partial_{x} U+\frac{\partial_{x} A}{A} U\right) \partial_{y} \bar{v} \\
& +\bar{v} \partial_{x} \bar{v}-\partial_{x} W(\bar{v}) \partial_{y} \bar{v}+\frac{\partial_{x} A}{A} W(\bar{v}) \partial_{y} \bar{v}+U \partial_{x} \bar{v}+\bar{v} \partial_{x} U=0 .
\end{aligned}
$$

We have made the change of variables $Y \mapsto y$ such that for a suitable choice of the function $A(x, t)$ we have

$$
\frac{\partial_{t} A}{A}-\partial_{x} U+\frac{\partial_{x} A}{A} U=0
$$

Indeed, letting $A(x, t)$ be the a solution of the initial value problem

$$
\begin{aligned}
& \partial_{t} A+U \partial_{x} A=A \partial_{x} U, \\
& A(x, 0)=1
\end{aligned}
$$

on $\mathbb{R} \times[0, T]$, we have that (A.18) holds, and therefore (A.17) becomes

$$
\partial_{t} \bar{v}-A^{2} \partial_{y y} \bar{v}+\bar{v} \partial_{x} \bar{v}-\partial_{x} W(\bar{v}) \partial_{y} \bar{v}+\frac{\partial_{x} A}{A} W(\bar{v}) \partial_{y} \bar{v}+U \partial_{x} \bar{v}+\bar{v} \partial_{x} U=0 .
$$

We note that the since $U(x, t)$ (and hence also $\partial_{x} U(x, t)$ ) is a real-analytic function, we obtain from the Cauchy-Kowalewski Theorem that if $T$ is sufficiently small, there exists a unique real-analytic solution $A(x, t)$ to (A.19)-(A.20). Using characteristics, one may solve for $A(x, t)$ explicitly in terms of the flow map associated to the transport 
equation $\partial_{t} A+U \partial_{x} A=0$, and it is not hard to check that by possibly reducing $T$ we have $A(x, t) \geq 1 / 2$ on $\mathbb{R} \times[0, T]$. The third change of variables

$$
v(x, y, t)=\bar{v}(x, y, t)+\phi(y) U(x, t),
$$

where $\phi(y)=1 /\langle y\rangle^{\theta}$ for some $\theta>0$, is made so that the boundary terms of $v$ at both $Y=0$ and $Y=\infty$ vanish, that is

$$
\left.v(x, y, t)\right|_{y=0}=0 \quad \text { and } \quad \lim _{y \rightarrow \infty} v(x, y, t)=0
$$

for all $(x, t) \in \mathbb{R} \times[0, T]$. Using (A.21), the evolution equation satisfied by $v$ is

$$
\begin{aligned}
\partial_{t} v- & \phi \partial_{t} U-A^{2}\left(\partial_{y y} v-\partial_{y y} \phi U\right) \\
& +(v-\phi U)\left(\partial_{x} v-\phi \partial_{x} U\right)-\left(\partial_{x} W(v)-\Phi \partial_{x} U\right)\left(\partial_{y} v-\partial_{y} \phi U\right) \\
& +\frac{\partial_{x} A}{A}(W(v)-\Phi U)\left(\partial_{y} v-\partial_{y} \phi U\right)+U\left(\partial_{x} v-\phi \partial_{x} U\right)+(v-\phi U) \partial_{x} U=0,
\end{aligned}
$$

which after a short computation may be rewritten as

$$
\partial_{t} v-A^{2} \partial_{y y} v+N(v)+L(v)=F,
$$

where

$$
\begin{aligned}
& \begin{array}{l}
N(v)=v \partial_{x} v-\partial_{x} W(v) \partial_{y} v+\partial_{x} a W(v) \partial_{y} v \\
L(v)=\partial_{x} W(v) \partial_{y} \phi U+\partial_{x} v(1-\phi) U \\
\quad+\partial_{y} v\left(\Phi \partial_{x} U-\frac{\partial_{x} A}{A} \Phi U\right)-W(v) \frac{\partial_{x} A}{A} \partial_{y} \phi U+v(1-\phi) \partial_{x} U \\
\quad F=\left(\phi(1-\phi)+\Phi \partial_{y} \phi\right) U \partial_{x} U-\frac{\partial_{x} A}{A} \partial_{y} \phi \Phi U^{2}-A^{2} \partial_{y y} \phi U-\phi \partial_{x} P,
\end{array}
\end{aligned}
$$

and $\Phi(y)=\int_{0}^{y} \phi(\zeta) d \zeta$

\section{REFERENCES}

[1] K. Asano, A note on the abstract Cauchy-Kowalewski theorem, Proc. Japan Acad. Ser. A, 64, 102-105, 1988.

[2] C. Bardos and S. Benachour, Domaine d'analycité des solutions de l'équation d'Euler dans un ouvert de $\mathbb{R}^{n}$, Ann. Scuola Norm. Sup. Pisa Cl. Sci., 4(4), 647-687, 1977.

[3] G.K. Batchelor, An Introduction to Fluid Dynamics, Cambridge Mathematical Library, Cambridge University Press, Cambridge, 1999.

[4] R.E. Cafflish and M. Sammartino, Existence and singularities for the Prandtl bounadry layer equations, Z. Angew. Math. Mech., 80, 733-744, 2000.

[5] P. Constantin and J. Wu, Inviscid limit for vortex patches, Nonlin., 8(5), 735-742, 1995.

[6] P. Constantin and J. Wu, The inviscid limit for non-smooth vorticity, Indiana U. Math. J., 45, 67-81, 1996.

[7] J. Cousteix and J. Mauss, Asymptotic Analysis and Boundary Layers, Sci. Comput. Springer, Berlin, 2007.

[8] L.L. van Dommelen and S.F. Shen, The spontaneous generation of the singularity in a separating laminar boundary layer, J. Comput. Phys., 38, 125-140, 1980.

[9] W. E, Boundary layer theory and the zero-viscosity limit of the Navier-Stokes equations, Acta Math. Sin., 16, 207-218, 2000.

[10] W. E and B. Engquist, Blowup of solutions of the unsteady Prandtl's equation, Commun. Pure Appl. Math., 50, 1287-1293, 1997.

[11] F. Gargano, M. Sammartino, and V. Sciacca, Singularity formation for Prandtl's equations, Physica D, 238, 1975-1991, 2009. 
[12] D. Gérard-Varet and E. Dormy, On the ill-posedness of the Prandtl equation, J. Amer. Math. Soc., 23, 591-609, 2010.

[13] D. Gérard-Varet and T. Nguyen, Remarks on the ill-posedness of the Prandtl equation, Asymp. Anal., 77, 77-88, 2012.

[14] E. Grenier, On the stability of boundary layers of incompressible Euler equations, J. Diff. Eqs., $164,180-222,2000$.

[15] E. Grenier, On the nonlinear instability of Euler and Prandtl equations, Commun. Pure Appl. Math., 53, 1067-1091, 2000.

[16] L. Hong and J.K. Hunter, Singularity formation and instability in the unsteady inviscid and viscous Prandtl equations, Commun. Math. Sci., 1(2), 293-316, 2003.

[17] T. Kato, Remarks on zero viscosity limit for nonstationary Navier-Stokes flows with boundary, MSRI Publ., Springer-Verlag, New York, 2, 8598, 1984.

[18] J.P. Kelliher, On Kato's conditions for vanishing viscosity, Indiana Univ. Math. J., 56, 4, 1711-1721, 2007.

[19] I. Kukavica, R. Temam, V. Vicol, and M. Ziane, Local existence and uniqueness for the hydrostatic Euler equations on a bounded domain, J. Diff. Eqs., 250, 1719-1746, 2011.

[20] I. Kukavica and V. Vicol, On the radius of analyticity of solutions to the three-dimensional Euler equations, Proc. Amer. Math. Soc., 137, 669-677, 2009.

[21] I. Kukavica and V. Vicol, The domain of analyticity of solutions to the three-dimensional Euler equations in a half space, Discrete Contin. Dyn. Syst., 29, 1, 285-303, 2011.

[22] I. Kukavica and V. Vicol, On the analyticity and Gevrey-class regularity up to the boundary for the Euler equations, Nonlin., 24, 765-796, 2011.

[23] M.C. Lombardo, M. Cannone, and M. Sammartino, Well-posedness of the boundary layer equations, SIAM J. Math. Anal., 35(4), 987-1004, 2003.

[24] M.C. Lopes Filho, A.L. Mazzucato, and H.J. Nussenzveig Lopes, Vanishing viscosity limit for incompressible flow inside a rotating circle, Phys. D, 237(10-12), 1324-1333, 2008.

[25] N. Masmoudi, The Euler limit of the Navier-Stokes equations, and rotating fluids with boundary, Arch. Rational Mech. Anal., 142(4), 375-394, 1998.

[26] A. Mazzucato and M. Taylor, Vanishing viscosity plane parallel channel flow and related singular perturbation problems, Anal. PDE, 1(1), 35-93, 2008.

[27] K. Nickel, Prandtl's boundary layer equations from the viewpoint of a mathematician, Ann. Rev. Fluid Mech., 5, 405-428, 1973.

[28] O.A. Oleinik, On the mathematical theory of boundary layer for an unsteady flow of incompressible fluid, J. Appl. Math. Mech., 30, 951-974, 1966.

[29] O.A. Oleinik and V.N. Samokhin, Mathematical models in boundary layer theory, Appl. Math. Math. Comput., Chapman \& Hall/CRC, Boca Raton, FL, 15, 1999.

[30] L. Prandtl, Über Flüssigkeitsbewegung bei sehr kleiner Reibung, Verh. III Intern. Math. Kongr. Heidelberg, Teuber, Leipzig, 485-491, 1904.

[31] M. Sammartino and R.E. Caflisch, Zero viscosity limit for analytic solutions of the NavierStokes equation on a half-space. I. Existence for Euler and Prandtl equations, Commun. Math. Phys., 192, 433-461, 1998.

[32] M. Sammartino and R.E. Caflisch, Zero viscosity limit for analytic solutions of the NavierStokes equation on a half-space. II. Construction of the Navier-Stokes solution, Commun. Math. Phys., 192, 463-491, 1998.

[33] H. Schlichting, Boundary Layer Theory, 4th ed. McGraw-Hill Series in Mechanical Engineering McGraw-Hill Book Co., Inc., New York-Toronto-London; Verlag G. Braun, Karlsruhe, 1960.

[34] R. Temam and X. Wang, The convergence of the solutions of the Navier-Stokes equations to that of the Euler equations, Appl. Math. Lett., 10(5), 29-33, 1997.

[35] R. Temam and X. Wang, Boundary layers associated with incompressible Navier-Stokes equations: The noncharacteristic boundary case, J. Diff. Eqs., 179(2), 647-686, 2002.

[36] W. Walter, On the asymptotic behavior of solutions of the Prandtl boundary layer equations, Indiana Univ. Math. J., 20, 829-841, 1970/1971.

[37] Z. Xin and L. Zhang, On the global existence of solutions to the Prandtl's system, Adv. Math., 181(1), 88-133, 2004. 